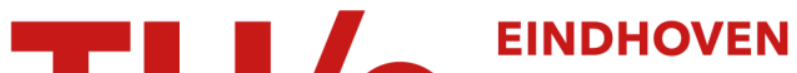 \\ UNIVERSITY OF \\ TECHNOLOGY
}

\section{Flow-regime analysis of non-Newtonian duct flows}

Citation for published version (APA):

Speetjens, M. F. M., Rudman, M., \& Metcalfe, G. (2006). Flow-regime analysis of non-Newtonian duct flows. Physics of Fluids, 18(1), 013101-1/14. https://doi.org/10.1063/1.2163913

DOI:

$10.1063 / 1.2163913$

Document status and date:

Published: 01/01/2006

\section{Document Version:}

Publisher's PDF, also known as Version of Record (includes final page, issue and volume numbers)

\section{Please check the document version of this publication:}

- A submitted manuscript is the version of the article upon submission and before peer-review. There can be important differences between the submitted version and the official published version of record. People interested in the research are advised to contact the author for the final version of the publication, or visit the $\mathrm{DOI}$ to the publisher's website.

- The final author version and the galley proof are versions of the publication after peer review.

- The final published version features the final layout of the paper including the volume, issue and page numbers.

Link to publication

\section{General rights}

Copyright and moral rights for the publications made accessible in the public portal are retained by the authors and/or other copyright owners and it is a condition of accessing publications that users recognise and abide by the legal requirements associated with these rights.

- Users may download and print one copy of any publication from the public portal for the purpose of private study or research.

- You may not further distribute the material or use it for any profit-making activity or commercial gain

- You may freely distribute the URL identifying the publication in the public portal.

If the publication is distributed under the terms of Article 25fa of the Dutch Copyright Act, indicated by the "Taverne" license above, please follow below link for the End User Agreement:

www.tue.nl/taverne

Take down policy

If you believe that this document breaches copyright please contact us at:

openaccess@tue.nl

providing details and we will investigate your claim. 


\title{
Flow regime analysis of non-Newtonian duct flows
}

\author{
Michel Speetjens, ${ }^{\text {a) }}$ Murray Rudman, and Guy Metcalfe \\ CSIRO Manufacturing and Infrastructure Technology, P.O. Box 56, Highett, VIC 3190, Australia
}

(Received 5 May 2005; accepted 20 October 2005; published online 23 January 2006)

\begin{abstract}
Reoriented duct flows of generalized Newtonian fluids are an idealization of non-Newtonian fluid flow in industrial in-line mixers. Based on scaling analysis and computation we find that non-Newtonian duct flows have several limit behaviors, in the sense that such flows can become (nearly) independent of one or more of the rheological and dynamical control parameters, simplifying the general flow and mixing problem. These limit flows give several levels of modeling complexity to the full problem of non-Newtonian duct flow. We describe the sets of simplified flow models and their corresponding regions of validity. This flow-model decomposition captures the essential rheological and dynamical characteristics of the reoriented duct flows and enables a more efficient and systematic study and design of flow and mixing of non-Newtonian fluids in ducts. Key aspects of the flow-model decomposition are demonstrated via a specific, but representative, duct flow. [DOI: 10.1063/1.2163913]
\end{abstract}

\section{INTRODUCTION}

Reoriented duct flows consist of a superposition of a uniaxial flow, a forcing of flow transverse to the throughflow direction, and a systematic reorientation of this transverse flow. Such duct flows are generically representative of continuous mixing flows and represent the fundamental working principle of continuous in-line mixers for viscous fluids. Examples of reoriented duct flows include the well-known Kenics mixer, the multiflux mixer, ${ }^{1}$ the Sulzer SMX mixer, ${ }^{2}$ and, a more recent development, the rotated arc mixer (RAM). ${ }^{3}$ Notwithstanding widespread application of this mixing principle in industry, still there is a limited understanding of its underlying mechanisms. Moreover, scientific studies on inline mixers are in general case specific and mainly restricted to Newtonian fluids. The Kenics mixer and an idealization thereof, the partitioned-pipe mixer, are the most widely studied configurations ${ }^{4-9}$; other configurations enjoy only occasional attention. ${ }^{10,11}$ Investigations involving non-Newtonian fluids are relatively scarce and as a rule confined to powerlaw fluids. ${ }^{3,11-13}$ A better, fundamental understanding of nonNewtonian duct flows would be useful for the design of mixers and heat exchangers for viscous fluids. Non-Newtonian flows are often obscured by their complexity ${ }^{14}$ and in this paper we show a scaling analysis on the generic rheological and dynamical behaviors of three-dimensional (3D) reoriented duct flows for generalized Newtonian fluids that reduces the obscuring complexity over large ranges of control parameter space.

Generalized Newtonian fluids model acceptably well a wide range of industrial flows having yield and pseudoplasticity. ${ }^{15}$ Specifically we will consider the Herschel-Bulkley rheology model, which incorporates both yield and pseudoplasticity; other rheological effects, such as elasticity, are ignored. For the analysis we will also assume

\footnotetext{
${ }^{a)}$ Present address: Institut für Geometrie und Praktische Mathematik, RWTH Aachen, Templergraben 55, D-52056 Aachen, Germany.
}

the flows are noninertial, which is a suitable assumption for viscous enough fluids. Further, we will assume that a reoriented, cellular flow model well approximates the flow reorientation that happens periodically along the duct. Experimentally validated for viscous fluids, ${ }^{3}$ we elaborate this assumption in Sec. II, but in essence it consists of taking as the basic flow one transverse and one axial flow and assuming that the full 3D duct flow is well approximated by reoriented copies of this base flow cell that are "bolted" together to approximate the full duct flow. A cellular flow model requires resolution of the 3D flow field within one cell only and substantially reduces computational effort compared to resolution of the full duct flow.

Over ranges of the control parameter space specific rheological and/or dynamical features of the flow field dominate, effectively reducing the full duct flow to limit flows with rheology and/or dynamics simpler than that of the full flow field. In such ranges, non-Newtonian behavior may become dominated by shear thinning or yielding only and/or be dependent upon the transverse or axial flow only. Limit behavior causes the non-Newtonian flow to have several levels of complexity, ranging from the full Herschel-Bulkley rheology down to the Newtonian baseline. At each level of reduced complexity the flow field collapses onto a limit flow that is described by a simplified form of the cellular flow model. This limit behavior decomposes the cellular flow model into a set of simplified flow models that each have a specific region of validity in parameter space. Such a flow-model decomposition offers a way to disclose generic rheological and dynamical properties of duct flows. The main advantage of a scaling analysis is that the results are universal in the sense of not depending on the specifics of the geometry and forcing. ${ }^{16}$ Moreover, the analysis enables simplification of the cellular flow model without compromising its physical validity.

The paper is organized as follows. Section II gives the problem definition, governing equations and control parameters. Limit behavior of the flow dynamics and the resulting 


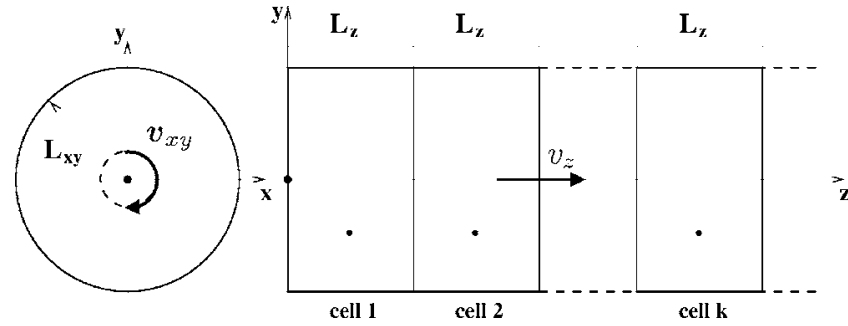

FIG. 1. The generic duct flow illustrated schematically by means of the circular case.

simplifications to the cellular flow model are considered in Sec. III. Section IV derives regions of validity for the limit model flows. The generic flow-model decomposition and its visual representation, the flow-model diagram, are specified in Sec. V. In Sec. VI we apply the flow-model decomposition to a specific, but representative, experimental duct flow. Conclusions are in Sec. VII.

\section{GENERALIZED NEWTONIAN DUCT FLOWS}

\section{A. Herschel-Bulkley rheology model}

The motion of generalized Newtonian fluids under noninertial and steady flow conditions is described by the Stokes equations

$$
\begin{aligned}
& \nabla \cdot \mathbf{v}=0, \quad \nabla P=\nabla \cdot \mathbf{T}, \quad \mathbf{T}=2 \alpha(\dot{\gamma}) \mathbf{D}, \\
& \dot{\gamma}=\sqrt{2 \mathbf{D}: \mathbf{D}}, \quad \mathbf{D}=1 / 2\left[\nabla \mathbf{v}+(\nabla \mathbf{v})^{T}\right],
\end{aligned}
$$

where $\mathbf{v}$ is the global velocity field, $P$ is the pressure, $\mathbf{T}$ is the stress tensor, and quantities $\alpha, \dot{\gamma}$, and $\mathbf{D}$ are the dynamic viscosity, shear rate, and the strain-rate tensor, respectively. The fluid rheology is modeled by the Herschel-Bulkley (HB) model,

$$
\alpha(\dot{\gamma})=\frac{\tau_{0}}{\dot{\gamma}}+\kappa \dot{\gamma}^{n-1},
$$

where $\tau_{0}$ is the yield stress, $\kappa$ is the fluid consistency, and $n$ is the flow index. With both yield $\left(\tau_{0}>0\right)$ and pseudo-plastic $(n \neq 1)$ effects, this model accounts for the principal rheological effects of generalized Newtonian fluids. As shear thinning is the most common pseudo-plastic behavior in applications, ${ }^{15}$ we will give results here only for $n<1$.

\section{B. Reoriented duct flows}

The flow domain consists of a duct of constant cross section (with characteristic transverse dimension $L_{x y}$ ) that is axially partitioned into a sequence of cells each of length $L_{z}$. Figure 1 gives a schematic of the flow configuration for the case of a circular cross section. The axial flow $v_{z}$ is steady and vanishes on the duct wall. ${ }^{17}$ The transverse flow $\mathbf{v}_{x y}$ $=\left(v_{x}, v_{y}\right)$ is subject to no-slip boundary conditions of the form $\mathbf{U}_{x y}\left(\theta-\theta_{R}(z)\right)$, where $\theta_{R}(z)=\Theta \sum_{k=1}^{\infty} \mathcal{H}\left(z-k L_{z}\right)$ is the reorientation angle and $\mathcal{H}$ is the Heaviside function. This accomplishes the systematic cell-wize reorientation of the basic transverse boundary conditions $\mathbf{U}_{x y}$ with incremental flow reorientation $\Theta$. Conditions $\mathbf{U}_{x y}$ represent some transverse forcing mechanism that may include internal elements. The form of $\mathbf{U}_{x y}$ requires the cross section to be invariant under the reorientation; further, we consider only convex cross sections, i.e., duct shapes are circles and regular polygons of $K$ edges, with $K$ integer multiples of $2 \pi / \Theta$.

\section{Cellular duct flow model}

Flow conditions are assumed such that a reoriented, cellular flow model well approximates the fluid motion. This cellular model hinges on two conditions: (i) duct flow with reorientation identical to that of the transverse forcing; (ii) negligible intercellular transient effects. 3D simulations on the Kenics mixer yield flow fields with reorientation synchronous to that of the transverse forcing, evidence of the general validity of the condition (i). ${ }^{4}$ The relative changeover length associated with intercellular transients diminishes for sufficiently-elongated cells, implying validity of condition (ii) depends primarily on geometric dimensions. Secondary effects that may magnify the relative changeover length are inertia and rheology. We have assumed a noninertial flow because Hobbs et al. ${ }^{4}$ found inertial effects in the Kenics mixer were insignificant up to $\mathrm{Re} \approx 10$ and Metcalfe et al. $^{3}$ found inertial effects in the RAM flow were insignificant up to $\operatorname{Re} \approx 50$. Rheology-induced transients at cell boundaries are more problematic. If the cellular flow model is a simple reduction to a plane cross section and does not account for all three velocity gradients, a cellular model can differ appreciably from experiment. The model below will properly account for all in-plane and axial velocities and gradients, so intercellular transients will be negligible for generalized Newtonian fluids; however, experiments show that moderately viscoelastic fluids (Deborah numbers $>1$ ) violate assumption (ii). ${ }^{3}$ These considerations suggest the two conditions hold true under the proviso of sufficiently elongated mixer cells, and the results below will apply to such duct flows.

Reorientation of the flow field identical to that of the transverse forcing means the reorientation of the transverse boundary condition, i.e., $\mathbf{U}_{x y}\left(\theta-\theta_{R}(z)\right)$, carries over to the internal flow and thus implies solutions of the form $\mathbf{S}(r, \theta, z)=\mathbf{s}\left(r, \theta-\theta_{R}(z), z\right)$, where $\mathbf{S}=(\mathbf{v}, P, \mathbf{T})$ is the global solution for the relevant quantities and $\mathbf{s}=(\mathbf{u}, p, \boldsymbol{\tau})$ is its underlying cellular solution. Neglect of intercellular transient effects further means that for $\mathbf{U}_{x y}=\mathbf{U}_{x y}(x, y)$ the flow field depends upon the transverse coordinates $(x, y)$ only, implying $\mathbf{u}=\mathbf{u}(x, y)$ and, in consequence, $p=\tilde{p}(x, y)+C_{p} z$, where $C_{p}$ is the constant axial pressure gradient, and $\boldsymbol{\tau}=\boldsymbol{\tau}(x, y)$. These considerations simplify the flow model (1) into the cellular duct flow model

$$
\begin{aligned}
& \nabla \cdot \mathbf{u}_{x y}=0, \quad \nabla \tilde{p}=\nabla \cdot\left(2 \alpha \mathbf{D}_{x y}\right), \\
& C_{p}=\nabla \cdot\left(\alpha \nabla u_{z}\right), \quad \mathbf{D}_{x y}=1 / 2\left[\nabla \mathbf{u}_{x y}+\left(\nabla \mathbf{u}_{x y}\right)^{T}\right],
\end{aligned}
$$

with henceforward $\nabla=(\partial / \partial x, \partial / \partial y)$, governing the cellular transverse $\left(\mathbf{u}_{x y}\right)$ and axial $\left(\mathbf{u}_{z}\right)$ flows in the cross section. (In the following, subscripts $x, y$, and $z$ refer to transverse and axial components, respectively, of cellular flow quantities.) The viscosity $\alpha$ remains as in (2), with $\dot{\gamma}$ as before and the 


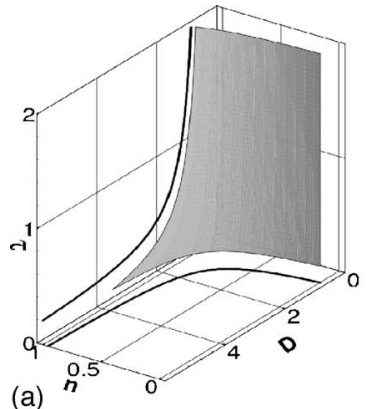

(a)

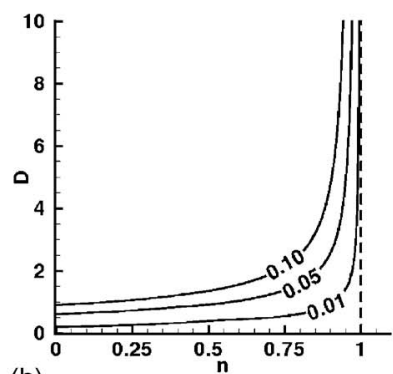

(b)

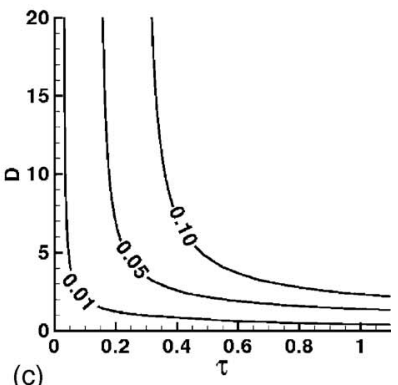

(c)
FIG. 2. Threshold $D_{\max }$ in the (a) $(n, \tau, D)$ space at $\epsilon=0.05$ and projected into the (b) $(n, D)$ plane and (c) $(\tau, D)$ plane for various $\epsilon$ as labeled. The dashed vertical line in (b) indicates the Newtonian limit. contraction of the strain-rate tensor reduced to $\mathbf{D}: \mathbf{D}$ $=\mathbf{D}_{x y}: \mathbf{D}_{x y}+(1 / 2)\left|\nabla u_{z}\right|^{2}$. The cellular model (3) is a reduction in space to a plane cross section of the duct with the crucial retention of all three velocity components and their gradients. ${ }^{18}$ We will refer to this as a $2.5 \mathrm{D}$ cellular model. ${ }^{19}$

\section{Nondimensional formulation and control parameters}

The scaling analysis hinges on a nondimensional formulation of the cellular model. We scale the momentum equation (3) by $\mathbf{x}_{x y}^{\prime}=\mathbf{x}_{x y} / L_{x y}, \mathbf{u}_{x y}^{\prime}=\mathbf{u}_{x y} / U_{x y}, u_{z}^{\prime}=u_{z} / U_{z}, \tilde{p}^{\prime}=\tilde{p} / \kappa c^{n}$, $C_{p}^{\prime}=C_{p} L_{x y} U_{x y} / \kappa c^{n} U_{z}$ and $\alpha^{\prime}=\alpha / \kappa c^{n-1}$, with $U_{x y}$ and $U_{z}$ the characteristic transverse and axial velocities, respectively, and $c=U_{x y} / L_{x y}$, yielding $\mathbf{D}^{\prime}=\mathbf{D} / c$ and $\dot{\gamma}^{\prime}=\dot{\gamma} / c$. Substitution into (3) and omitting primes gives the nondimensional momentum equation. The corresponding nondimensional HB model (upon dropping primes) is

$$
\begin{aligned}
& \alpha(\dot{\gamma})=\frac{\tau}{\dot{\gamma}}+\dot{\gamma}^{n-1}, \quad \dot{\gamma}=\sqrt{2 \mathbf{D}: \mathbf{D},} \\
& \mathbf{D}: \mathbf{D}=\mathbf{D}_{x y}: \mathbf{D}_{x y}+\frac{D^{2}}{2}\left|\nabla u_{z}\right|^{2} .
\end{aligned}
$$

The control parameters are the nondimensional yield stress $\tau=\tau_{0} / \kappa c^{n}$ (also known as the Bingham number), the flow index $n$ and flow ratio $D=U_{z} / U_{x y}$. The scaling places all the control parameters into the viscosity $\alpha$. Note that the different scalings for transverse and axial velocities means the actual nondimensional velocity fields, in terms of $U_{x y}$, read $\mathbf{u}=\left(u_{x}, u_{y}, D u_{z}\right)$ and $\mathbf{v}=\left(v_{x}, v_{y}, D v_{z}\right)$.

\section{LIMIT BEHAVIOR AND PRINCIPAL SIMPLIFICATIONS}

The cellular flow model exhibits limit behavior in the sense that it becomes independent of one or more of the governing parameters and reduces to simpler forms in specific regions of its $3 \mathrm{D}$ parameter space $\mathcal{S}:[n, \tau, D]$. In such regions the flow field collapses onto limit flows that are described by simpler forms of the cellular model. Each limit flow in turn has a specific region of validity $(\mathrm{ROV})$ in $\mathcal{S}$.

It is preferable to obtain the limit flows and their ROVs as functions of the control parameters in a way independent of the particulars of forcing and geometry, and a universal scaling analysis of the physical flow accomplishes this. ${ }^{16}$ Which flow quantity should be used for the scaling analysis? Since every flow quantity belongs to the same flow field and behaves consistently, the specific choice is immaterial: universal scaling properties will be qualitatively similar for every choice. As all the control parameters for the cellular flow model are in the viscosity via Eq. (4), a scaling analysis on $\alpha$ is a natural way to obtain the needed decomposition of limit flows and their trends and changes as parameters are varied. In Sec. VI we will find it convenient to analyze the velocity and will explicitly show the similarity of results.

There are two principal kinds of simplifications: rheological simplifications and dynamical simplifications. Rheological parameters $\tau$ and $n$ control the contribution of shear thinning and yielding to $\alpha$. Dynamical parameter $D$ controls the relative contribution of the transverse and axial flows to the shear rate $\dot{\gamma}$. The fundamental flow states of the duct flows result from a given combination of one simplified rheological state and one simplified dynamical state and are described by the flow-model decomposition. The remainder of this section takes the limits and discusses the physical meaning of the simplified rheological and dynamical nonNewtonian flows, after which the approximate ROVs of the limits are calculated. This enables the generic flow-model decomposition and construction of flow-model diagrams that can be used to analyze and design non-Newtonian duct flows.

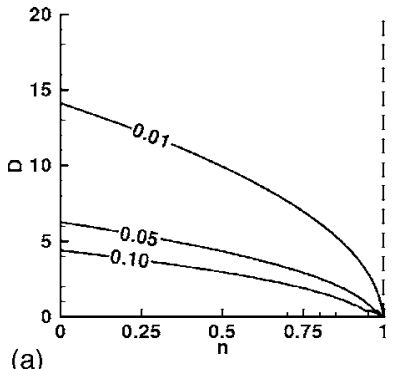

(a)

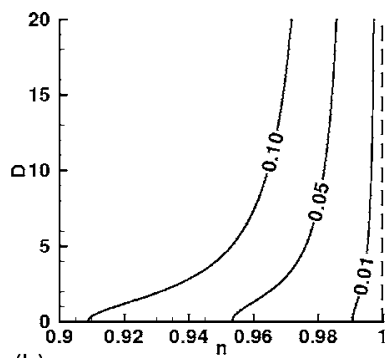

(b)

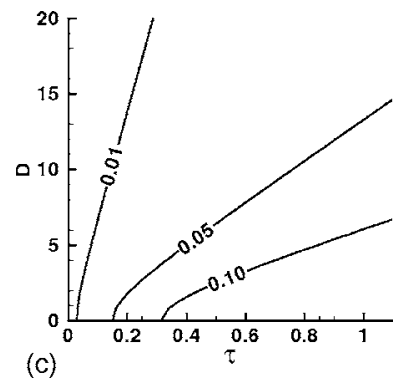

(c)
FIG. 3. Intersections of (a) $D_{\min }$ and (b) $n_{\min }$ with the $(n, D)$ plane, and (c) $\tau_{\max }$ with the $(\tau, D)$ plane for various $\epsilon$. The dashed vertical line in (a) and (b) indicates the Newtonian limit $n$ $=1$. The contour labels are the tolerances $\epsilon$. 
TABLE I. Ten fundamental states of 2.5D duct flows. Each of these states results from a combination of one rheological state (columns) and one dynamical state (rows) of the flow field. The corresponding rheology models and associated ROVs are as indicated.

\begin{tabular}{|c|c|c|c|c|}
\hline \multirow[b]{2}{*}{ Dynamical states } & \multicolumn{4}{|c|}{ Rheological states } \\
\hline & Herschel-Bulkley & Power-law & Bingham & Newtonian \\
\hline \multirow[t]{3}{*}{ Transverse } & Model: $\alpha=\tau / \dot{\gamma}_{x y}+\dot{\gamma}_{x y}^{n-1}$ & Model: $\alpha=\dot{\gamma}_{x y}^{n-1}$ & Model: $\alpha=\tau / \dot{\gamma}_{x y}+1$ & \\
\hline & ROV: $D \leqslant D_{\max }$ & ROV: $D \leqslant D_{\max } \cup \tau \leqslant \tau_{\max }$ & ROV: $D \leqslant D_{\max } \cup n \geqslant n_{\min }$ & \\
\hline & Reference: $\alpha_{x y}$ & Reference: $\alpha_{x y, P}$ & Reference: $\alpha_{x y, B}$ & \\
\hline \multirow[t]{3}{*}{ Full } & Model: $\alpha=\tau / \dot{\gamma}+\dot{\gamma}^{n-1}$ & Model: $\alpha=\dot{\gamma}^{n-1}$ & Model: $\alpha=\tau / \dot{\gamma}+1$ & Model: $\boldsymbol{\alpha}=\mathbf{1}$ \\
\hline & $\mathrm{ROV}: \forall n, \tau, D$ & $\mathrm{ROV}: \tau \leqslant \tau_{\max }$ & ROV: $n \geqslant n_{\min }$ & $\mathrm{ROV}: \tau \leqslant \tau_{\max } \cup n \geqslant n_{\min }$ \\
\hline & Reference: $\alpha$ & Reference: $\alpha_{P}$ & Reference: $\alpha_{B}$ & Reference: $\alpha_{N}$ \\
\hline \multirow[t]{3}{*}{ Axial } & Model: $\alpha=\tau / \dot{\gamma}_{z}+\dot{\gamma}_{z}^{n-1}$ & Model: $\alpha=\dot{\gamma}_{z}^{n-1}$ & Model: $\alpha=\tau / \dot{\gamma}_{z}+1$ & \\
\hline & ROV: $D \geqslant D_{\min }$ & ROV: $D \geqslant D_{\min } \cup \tau \leqslant \tau_{\max }$ & ROV: $D \geqslant D_{\min } \cup n \leqslant n_{\min }$ & \\
\hline & Reference: $\alpha_{z}$ & Reference: $\alpha_{z, P}$ & Reference: $\alpha_{z, B}$ & \\
\hline
\end{tabular}

\section{A. Rheological simplification: Limit behavior of $\tau$ and $n$} limits

The rheological properties $\tau$ and $n$ have the asymptotic

$$
\lim _{\tau \rightarrow 0} \alpha=\dot{\gamma}^{n-1} \equiv \alpha_{P}, \quad \lim _{n \rightarrow 1} \quad \alpha=\frac{\tau}{\dot{\gamma}}+1 \equiv \alpha_{B},
$$

$\lim \lim \alpha \equiv \alpha_{N}=1$,

$$
\tau \rightarrow 0 n \rightarrow 1
$$

with the subscripts referring to the power-law $(P)$, Bingham $(B)$ and Newtonian $(N)$ fluid models. For completeness we remind the reader that for vanishing yield stress $\tau$ or for flow index $n$ approaching unity, the two-parameter HB model (4) simplifies to the one-parameter power-law or Bingham models, respectively. The ROVs for these one-parameter models are, strictly, the planes $\tau=0(P)$ and $n=1(B)$ in parameter space $\mathcal{S}$. The Newtonian limit $\alpha_{N}$ holds in the region where both $\tau$ and $n$ approach their respective limits.

In the Newtonian limit, constant $\alpha$ decouples the transverse and axial flows and leads to a velocity field of the form

$$
\mathbf{u}(\mathbf{x})=\left(u_{x}(\mathbf{x}), u_{y}(\mathbf{x}), D u_{z}(\mathbf{x})\right),
$$

in which $\tau$ and $n$ vanish altogether and $D$ is a multiplication factor to $u_{z}$. This implies that for given boundary conditions the Newtonian limit has a unique transverse flow and a selfsimilar axial flow with scaling factor $D$.

\section{B. Dynamical simplification: Limit behavior of $D$}

The limit behavior of $D$ is found in

$$
\lim _{D \rightarrow 0} \dot{\gamma}=\sqrt{2 \mathbf{D}_{x y}: \mathbf{D}_{x y}} \equiv \dot{\gamma}_{x y}, \quad \lim _{D \rightarrow \infty} \dot{\gamma}=D\left|\nabla u_{z}\right| \equiv \dot{\gamma}_{z},
$$

for which $\alpha$ reduces to

$$
\lim _{D \rightarrow 0} \alpha(\dot{\gamma})=\alpha\left(\dot{\gamma}_{x y}\right) \equiv \alpha_{x y}, \quad \lim _{D \rightarrow \infty} \alpha(\dot{\gamma})=\alpha\left(\dot{\gamma}_{z}\right) \equiv \alpha_{z} .
$$

These are limits for which non-Newtonian effects depend either only on the transverse $(D \rightarrow 0)$ or only on the axial $(D \rightarrow \infty)$ flow.

In the limit $D \rightarrow 0$ the viscosity depends solely on $\mathbf{u}_{x y}$, meaning the transverse and axial flows decouple and, due to fixed boundary conditions, implying $\mathbf{u}_{x y}$ is unique for a given rheology. This, in turn, implies a unique $\alpha=\alpha_{x y}$ and $u_{z}$, resulting again in a self-similar velocity field of the form (6) for constant $\tau$ and $n$. In the limit $D \rightarrow \infty$ the viscosity depends only on $u_{z}$ and again the transverse and axial flows decouple. Combining (4) with (7) and (8) gives (via l'Hôpital's rule) $\alpha_{z}=n \gamma_{z}^{n-1}=n D^{n-1}\left|\nabla u_{z}\right|^{n-1}$. Substituting this into the 2.5D flow model (4) gives

$$
\nabla p^{*}=\nabla \cdot\left(2 \alpha_{z, P} \boldsymbol{D}_{x y}\right), \quad C_{p}^{*}=\nabla \cdot\left(\alpha_{z, P} \nabla u_{z}\right),
$$

with $p^{*}=\tilde{p} / n, C_{p}^{*}=C_{p} / n$, and $\alpha_{z, P}=\dot{\gamma}_{z}^{n-1}$. By rescaling the pressure with $n$ the flow model collapses onto that for a power-law fluid-meaning yield effects become insignificant in the limit. However, this is a singular limit, and, as shown in Sec. V, with a finite region of validity yield effects remain. Equations (9) admit, just as for the limit $D \rightarrow 0$, self-similar solutions of the form (6) for a given fluid rheology. The associated ROVs are, naturally, the planes $D=0$ and $D \rightarrow \infty$.

\section{FINITE REGIONS OF VALIDITY FOR THE PRINCIPAL SIMPLIFICATIONS}

The analysis in Sec. III revealed that limit behavior causes five principal simplifications to the $2.5 \mathrm{D}$ model: rheological simplifications (5) and dynamical simplifications (8). True limit behavior requires (some) parameters to be infinitesimally close to their limit value for strict validity of the corresponding simplified flow model. However, a flowmodel decomposition based on strict bounds is of little use. The question of interest is: for a specified tolerance what is the finite region of validity in which the limit behavior dominates? Different tolerances will be appropriate for different end uses, e.g., rate-limited reactions versus blending for visual homogeneity. Allowing approximate limit behavior by tolerating finite departures from the cellular model extends (approximate) validity of simpler forms to finite parameter ranges. For such approximate limit behavior theoretical limits (5) and (8) relax to

$$
\begin{array}{ll}
\alpha=\alpha_{P} & \text { for } \tau \leqslant \tau_{\max }, \quad \alpha=\alpha_{B} \quad \text { for } n \geqslant n_{\min }, \\
\alpha=\alpha_{N} & \text { for } \tau \leqslant \tau_{\max } \cap n \geqslant n_{\min },
\end{array}
$$



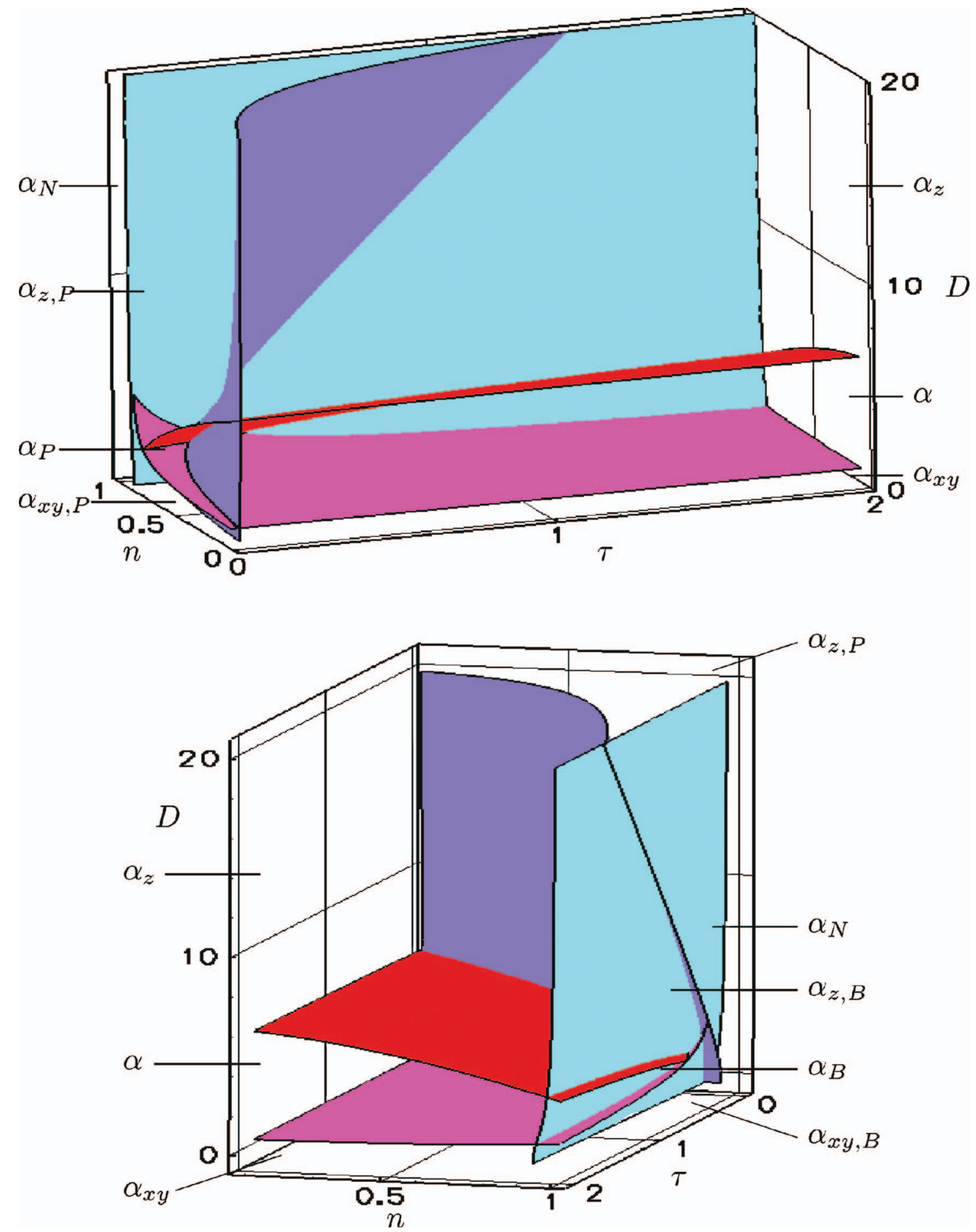

FIG. 4. (Color) Flow-model diagram for the HB model at tolerance $\epsilon=0.05$.

$$
\alpha=\alpha_{x y} \quad \text { for } D \leqslant D_{\max }, \quad \alpha=\alpha_{z} \quad \text { for } D \geqslant D_{\min } .
$$

Each of these regions defines the ROV of its corresponding simplified fluid model under these relaxed conditions and are natural extensions of the underlying theoretical ROVs of Sec. III. The bounds $\left(\tau_{\max }, n_{\min }, D_{\min }, D_{\max }\right)$ of the ROVs in (10) are the thresholds beyond which the difference between the full model and the simplified model exceeds a tolerance $\epsilon$. These thresholds are defined implicitly via

$$
\begin{aligned}
& \epsilon^{(P)}\left(\tau_{\max }\right)=\epsilon, \quad \epsilon^{(B)}\left(n_{\min }\right)=\epsilon, \\
& \epsilon^{(x y)}\left(D_{\max }\right)=\epsilon, \quad \epsilon^{(z)}\left(D_{\min }\right)=\epsilon,
\end{aligned}
$$

with $\epsilon^{(i)}=\left|\alpha_{i}-\alpha\right| /|\alpha|$ the relative departure from a given limit and $i \in[x y, z, P, B]$.

Expression of thresholds $\left(\tau_{\max }, n_{\min }, D_{\min }, D_{\max }\right)$ in terms of the control parameters provides the generic descriptions of 

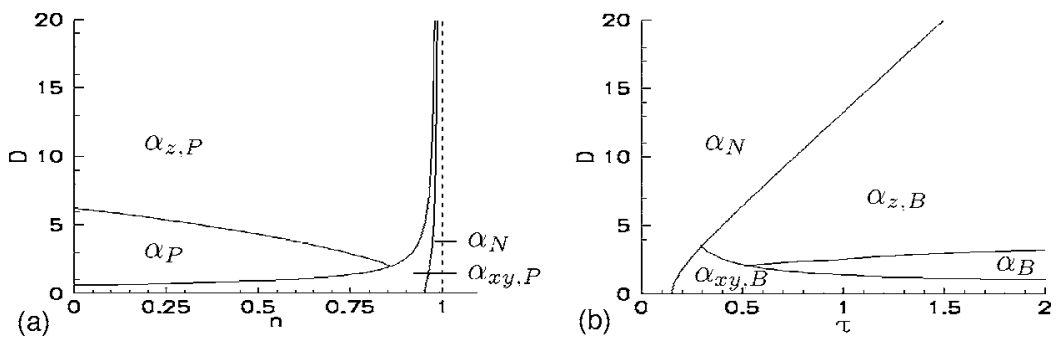

FIG. 5. Flow-model diagrams for (a) the power-law model and (b) the Bingham model at tolerance $\epsilon$ $=0.05$.

the ROVs. These expressions follow from the velocity gradients in the nondimensional contraction ${ }^{20}$ of $\mathbf{D}$ being all of $\mathcal{O}(1)$. Thus the shear relation $\dot{\gamma}=\sqrt{2 \mathbf{D}: \mathbf{D}}$ leads to

$$
\dot{\bar{\gamma}}=\sqrt{8+2 D^{2}}, \quad \dot{\bar{\gamma}}_{x y}=\sqrt{8}, \quad \dot{\bar{\gamma}}_{z}=\sqrt{2} D,
$$

as order-of-magnitude estimates for the nondimensional shear rates. Substitution of (12) in fluid model (4) yields scaling relations of the form $\bar{\alpha}=\bar{\alpha}(D, \tau, n)$ for the (simplified) viscosities. Substitution of the latter into (11), after rearrangement, gives

$$
\begin{aligned}
& \tau_{\max }(n, D)=\frac{\epsilon}{1-\epsilon}\left[8+2 D^{2}\right]^{n / 2}, \\
& n_{\min }(\tau, D)=\frac{\ln (\dot{\bar{\gamma}}-\epsilon \tau)-\ln (\epsilon+1)}{\ln \dot{\bar{\gamma}}}, \\
& H\left(D_{\max }(n, \tau)\right)=0, \quad H=\tau+\dot{\bar{\gamma}}^{n}-A \dot{\bar{\gamma}}, \\
& A=\frac{\tau+8^{n / 2}}{\sqrt{8}(\epsilon+1)}, \quad G\left(D_{\min }(n, \tau)\right)=0, \\
& G=\tau \dot{\bar{\gamma}}-\tau(\epsilon+1) \dot{\bar{\gamma}}_{z}+\dot{\bar{\gamma}}_{z}^{n} \dot{\bar{\gamma}}^{-}(\epsilon+1) \dot{\bar{\gamma}}_{z} \dot{\bar{\gamma}}^{n},
\end{aligned}
$$

as (implicit) expressions for the thresholds in terms of the control parameters. These are the universal scaling relations that describe the limit behavior of generic duct flows.

What tolerance should be chosen? Although the universal scaling properties are unaffected by choice of tolerance, to present graphical results a specific value must be chosen and, in general, the choice will depend on the use intended for the analysis. Here we will chose $\epsilon=0.05$, based on results for the stability of Lagrangian mixing structures. ${ }^{21}$

Figure 2 shows (a) $D_{\max }$ in the 3D parameter space for $\epsilon=0.05$ and its intersections (heavy curves) with the (b) $(n, D)$ plane and $(\mathrm{c})(\tau, D)$ plane for several $\epsilon$. The region $0 \leqslant D \leqslant D_{\max }$ demarcates the ROV of the $D \rightarrow 0$ approximation to the cellular flow model. This region expands monotonically with increasing tolerance $\epsilon$ and is bounded by the vertical asymptote $\lim _{\tau \rightarrow \sqrt{8} \epsilon} \lim _{n \rightarrow 1} D_{\text {max }} \rightarrow \infty$ and the horizontal asymptote $\lim _{\tau \rightarrow \infty} D_{\max }=\lim _{n \rightarrow 0} D_{\max }=2 \sqrt{\epsilon(\epsilon+2)}$. This monotonic behavior increasingly restricts the ROV of the $D \rightarrow 0$ approximation the more pronounced the nonNewtonian nature of the fluid. The remaining thresholds $\left(n_{\min }, D_{\min }, \tau_{\max }\right)$ are shown in Fig. 3. Given are the intersections of $D_{\min }$ [Fig. 3(a)] and $n_{\min }$ [Fig. 3(b)] with the $(n, D)$ plane and the intersection of $\tau_{\max }$ with the $(\tau, D)$ plane [Fig. 3(c)] at various $\epsilon$. Regions $n \geqslant n_{\min }$ and $\tau \leqslant \tau_{\max }$ are the
ROVs of the Bingham and power-law approximations, respectively; region $D \geqslant D_{\text {min }}$ is the ROV of the $D \rightarrow \infty$ approximation. These ROVs have properties qualitatively similar to that of the ROV defined by $D_{\max }$ in that they expand monotonically with increasing $\epsilon$ and become increasingly restricted the stronger the non-Newtonian behavior. In line with intuition the need to resolve the full cellular flow model increases as the fluid rheology becomes more nonNewtonian.

\section{FLOW-MODEL DECOMPOSITION AND FLOW- MODEL DIAGRAMS}

Along with their ROVs the limit flows described previously decompose the duct flow of generalized Newtonian fluids into the ten fundamental flows given in Table I. These fundamental flows depend on the rheological and dynamical parameters $\tau, n$, and $D$. The rheological states in the table change according to the relative contribution of shear thinning and yielding to the total stress. The dynamical states change according to the relative share of transverse and axial shear to the total stress.

Combined into one graph, the ROVs define a flowmodel diagram that provides a visual representation of the flow states/regimes and the flow-model decomposition. An example diagram for $\epsilon=0.05$ is shown in Fig. 4. The flow regimes are demarcated by the four principal surfaces delineating the ROVs described by (13): $\tau_{\max }$ (blue); $n_{\min }$ (cyan); $D_{\max }$ (purple); $D_{\text {min }}$ (red). Surfaces $\tau_{\max }$ and $n_{\min }$ separate the rheological states $(\mathrm{HB}, \mathrm{P}, \mathrm{B}$, and $\mathrm{N})$; surfaces $D_{\min }$ and $D_{\max }$ separate the dynamical states (transverse, full, and axial) in the essentially non-Newtonian part of parameter space.

Interpretation of the flow-model diagram is enhanced through isoplane representations in terms of $\tau$ and $n$. Figure 5 demonstrates this for planes (a) $\tau=0$ and (b) $n=1$, defining

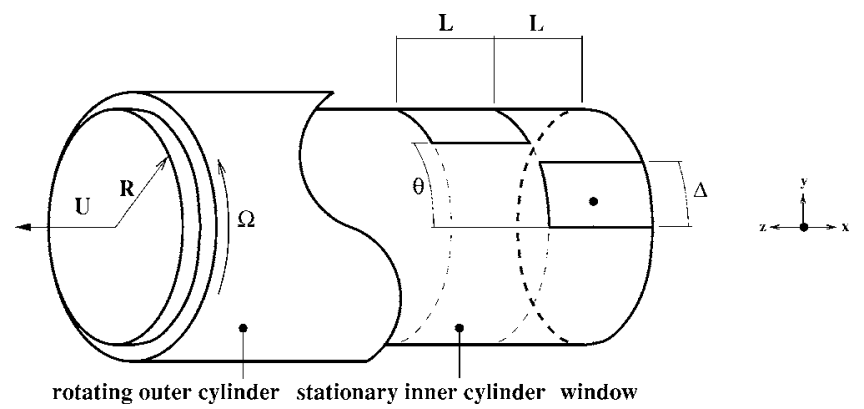

FIG. 6. Schematic of the rotated arc mixer flow. The test computations fix $\Delta=\pi / 4$ and for the analysis of this article a specific value for $\Theta$ is irrelevant. 


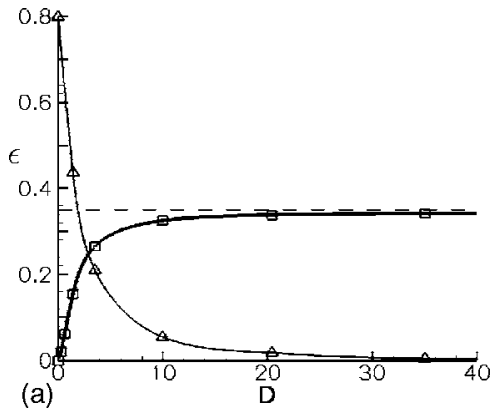

(a)

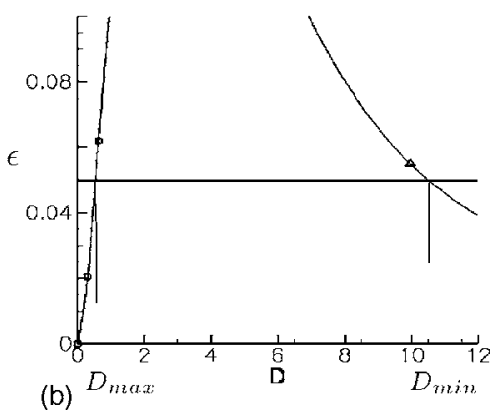

FIG. 7. Departures $\epsilon^{(x y)}$ (heavy) and $\epsilon^{(z)}$ (solid) from the limits $D \rightarrow 0$ and $D \rightarrow \infty$, respectively, at $n=0.5$, shown in (a) full view and in (b) close-up view. The profiles are smooth fits through numerical data (symbols). The dashed line in (a) is the horizontal asymptote $\epsilon_{\infty}^{(x y)}$; the horizontal line in (b) is the tolerance $\epsilon$ that intersects the profiles to give the thresholds $D_{\max }$ and $D_{\min }$. the flow-model diagrams for the power-law and Bingham models, respectively. Other isoplane plots are in the appendix. In both cases the ROV for the full cellular model expands under increasing non-Newtonian behavior. This suggests shear thinning and yield effects have a qualitatively similar influence on the flow. An essential difference, however, is that in the power-law limit the Newtonian regime contracts with increasing $D$, whereas it expands in the Bingham limit.

We stress the essential difference between true and approximate limit behavior in the limit $D \rightarrow \infty$. For true limit behavior yield effects become insignificant in the limit $D$ $\rightarrow \infty$ (see Sec. VI B) because of unbounded $D$ and in consequence the flow field simplifies to the self-similar form (6) in the corresponding theoretical ROV. However, for approximate limit behavior the limit $D \rightarrow \infty$ has a finite ROV including all of the bottom row of Table I in which yield effects remain significant in the sub-ROVs $\alpha_{z}$ and $\alpha_{z, B}$. This implies the self-similar form (6) of the velocity field holds only in the sub-ROV $\alpha_{z, P}$ here.

\section{FLOW-MODEL DECOMPOSITION APPLIED TO A REPRESENTATIVE DUCT FLOW}

Application of the flow-model decomposition is demonstrated for the RAM flow, which is representative of duct flows. ${ }^{3}$ This enabled, first, testing of the flow-model decomposition predicted by the viscosity-scaling analysis and, second, demonstration of typical flow patterns associated with this flow-model decomposition.

The RAM, shown schematically in Fig. 6, consists of two concentric snugly fitting cylinders. The stationary inner cylinder contains a series of windows via which the rotating outer cylinder sets up a transverse flow through viscous drag. These windows are periodically offset azimuthally in the axial direction to systematically reorient the transverse flow. With a steady axial flow the RAM creates a reoriented duct flow.

The RAM connects with the generic duct flow via $L_{x y}$ $=R, L_{z}=L, U_{x y}=\bar{\Omega} R$, and $U_{z}=U$, where $\bar{\Omega}$ and $U$ are the mean angular and axial velocities, respectively; $(D$ $=U /(\bar{\Omega} R), \tau, n)$ are the control parameters. The flow field is determined by analytical solutions ${ }^{22}$ and, if not available, by numerical simulations with the commercial finite-volume package CFX-5 (AEA Technology, UK). Solutions to the cellular flow model (3) are (for the noninertial assumption) always symmetric about the axis of symmetry $\theta=\Delta / 2$ of the transverse forcing, irrespective of fluid rheology. On this axis the velocity field (in polar coordinates) reduces to $\mathbf{u}$ $=\left(0, u_{\theta}, u_{z}\right)$. Plots of the flow quantities are, unless indicated otherwise, in the following given on this symmetry axis.

\section{A. Flow-model diagram for power-law fluids}

The generic flow-model decomposition is tested by directly calculating the flow-model diagram for the power-law case. This scaling analysis will be based on the velocity $\mathbf{u}$ rather than on the viscosity $\alpha$ (as in the previous analysis). Primarily this will demonstrate that the analysis underlying the generic flow-model decomposition may employ any flow quantity (see Sec. III), but it is also more convenient for application to viscous mixing problems (the properties of which depend primarily on the velocity). The thresholds $D_{\text {min }}, D_{\text {max }}$ and $n_{\min }$ corresponding with the power-law case are identified in a manner essentially similar to the approach adopted in the viscosity-scaling analysis (Sec. IV). Use

$$
\epsilon^{(i)}=\frac{L_{1}\left(\mathbf{u}-\mathbf{u}^{(i)}\right)}{L_{1}(\mathbf{u})}, \quad i \in[x y, z, N],
$$

analogous to relation (11). Here $\epsilon^{(i)}$ is the departure of the velocity $\mathbf{u}$ from its $D \rightarrow 0$ limit $\left(\epsilon^{(x y)}\right)$, its $D \rightarrow \infty \operatorname{limit}\left(\epsilon^{(z)}\right)$ and its Newtonian limit $\left(\epsilon^{(N)}\right)$, expressed in terms of the $L_{1}$-norm. ${ }^{25}$ Similar to (11), specifying $\epsilon^{(i)}$ in (14) implicitly defines the ROV thresholds. By way of example we calculate $D_{\min }$ and $D_{\max }$.

A robust procedure for determination of $D_{\max }$ and $D_{\min }$ begins by evaluating the departures from the corresponding limits $D \rightarrow 0$ (departure $\epsilon^{(x y)}$ ) and $D \rightarrow \infty$ (departure $\epsilon^{(z)}$ ), respectively. Figure 7(a) shows $\epsilon^{(x y)}$ (heavy) and $\epsilon^{(z)}$ (solid) as a function of $D$ for $n=0.5$. For increasing $D$ both profiles
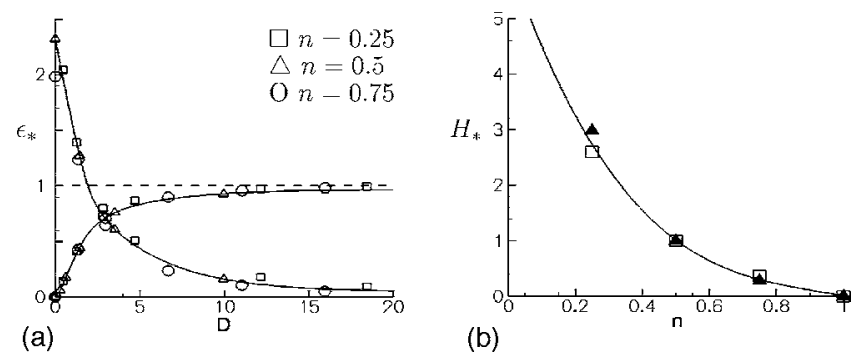

(b)

FIG. 8. (a) Self-similarity of departures $\epsilon^{(x y)}$ and $\epsilon^{(z)}$ and (b) the associated universal scaling function $H_{*}(n)$ for $n_{*}=0.5$. The curves in (a) are the normalized profiles $\epsilon_{*}^{(x y)}$ and $\epsilon_{*}^{(z)}$ at $n=0.5$; the symbols are the numerical data for $\epsilon_{*}^{(x y)}$ and $\epsilon_{*}^{(z)}$ at $n=[0.25,0.5,0.75]$. 


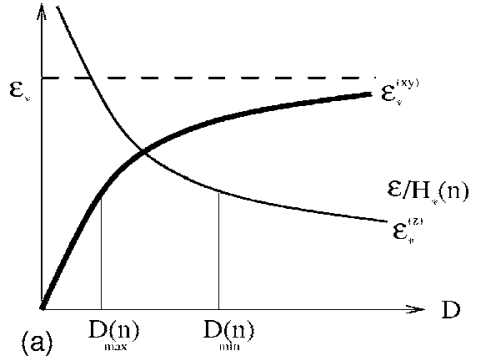

(a)

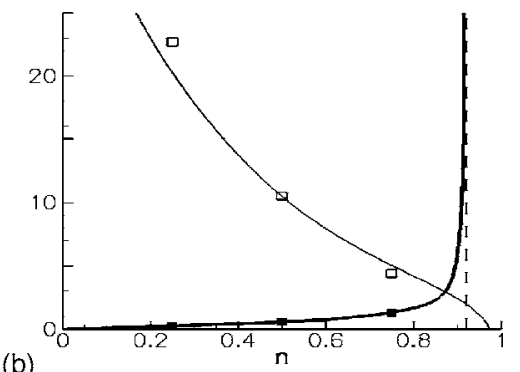

(b)

FIG. 9. (a) Relation departures $\epsilon_{*}^{(x y, z)}$ and thresholds $D_{[\min , \max ]}$ and (b) thresholds $D_{\max }$ (heavy) and $D_{\min }$ (solid) compared with direct calculations (symbols) for $\epsilon=0.05$. smoothly evolve at an equal rate from their starting values to their horizontal asymptotes and show the transition of $\mathbf{u}$ from its $D \rightarrow 0$ state to its $D \rightarrow \infty$ state. Figure 7(b) gives a closeup, in which the horizontal line is the tolerance $\epsilon$ that intersects the profiles, defining the thresholds $D_{\max }$ and $D_{\min }$. For any flow index $D_{\max }$ and $D_{\min }$ can be read from the corresponding $D-\epsilon^{(x y, z)}$ profiles. The threshold $n_{\min }$ is similarly determined from $n-\epsilon^{(N)}$ profiles.

The previous evaluation procedure for the thresholds can be improved by using the scaling properties of the associated departures. Figure 8(a) shows the normalized departures $\epsilon_{*}^{(x y, z)}(n)=\epsilon^{(x y, z)}(n) / \epsilon_{\infty}^{(x y)}(n)$, with $\epsilon_{\infty}^{(x y)}(n)$ the asymptotic value of $\epsilon^{(x y)}(n)$ [dashed line in Fig. 7(a)] for $n$ $=[0.25,0.5,0.75]$. The plot shows that the normalized data (symbols) for all $n$ collapse onto one curve for each $i$ $=x y, z$. This suggests the departures are self-similar in $n$. Self-similarity in turn implies the departures have the form

$\epsilon^{(i)}(n, D)=H_{*}(n) \epsilon_{*}^{(i)}(D), \quad \epsilon_{*}^{(i)}(D)=\epsilon^{(i)}\left(n_{*}, D\right), \quad i=x y, z$,

where $H_{*}(n)$ is a scaling function that has $H_{*}\left(n_{*}\right)=1$ and monotonically increases from $H_{*}(1)=0$, and where $\epsilon_{*}^{(i)}(D)$ and $n_{*}$ are reference values of departure and flow index, respectively. Further, the self-similarity has the important physical implication that the flow field is qualitatively similar over the considered range of the flow index $n$. This means that the flow field, despite perhaps substantial quantitative variation, does not undergo fundamental topological changes. ${ }^{16}$ Here $n_{*}=0.5$ is chosen, meaning $\epsilon_{*}^{(x y)}(D)$ and $\epsilon_{*}^{(z)}$ $\times(D)$ coincide with the departures shown in Fig. 7(a). The corresponding scaling function $H_{*}(n)$ is given in Fig. 8(b), where the profile is a fit through the numerical data (symbols). ${ }^{26}$

The scaling (15) can be used to determine the thresholds $D_{\text {[max,min }]}$ by collapsing the data for all $n$ (similarly to that in Fig. 7 for $n=0.5$ ) onto the universal curve of Fig. 9(a), where the profiles are the reference departures $\epsilon_{*}^{(x y)}(D)$ (heavy) and
$\epsilon_{*}^{(z)}(D)$ (solid) and the horizontal line is the rescaled tolerance $\epsilon_{*}(n)=\epsilon / H_{*}(n)$. The intersections $\epsilon_{*}^{(x y)}\left(D_{\max }(n)\right)=\epsilon / H_{*}(n)$ and $\epsilon_{*}^{(z)}\left(D_{\max }(n)\right)=\epsilon / H_{*}(n)$ implicitly define the thresholds at any flow index $n$. In fact the thresholds are mappings of the corresponding reference departures, i.e.,

$$
D_{\max }(n)=\epsilon_{*}^{(x y)^{-1}}\left(\epsilon / H_{*}(n)\right), \quad D_{\min }(n)=\epsilon_{*}^{(z)^{-1}}\left(\epsilon / H_{*}(n)\right),
$$

which determines thresholds $D_{\min }$ and $D_{\max }$ for all $n$ from the reference departures $\epsilon_{*}^{(x y, z)}$. This ability to utilize the selfsimilarity provides a significant reduction in cost compared to separate calculation for every $n$; one only needs to calculate for one $n$ to generate the reference curves, e.g., Fig.9. Figure 9(b) shows $D_{\max }$ (heavy) and $D_{\min }$ (solid) obtained via (16) in comparison to directly computed values (symbols) at $n=[0.25,0.5,0.75]$ for $\epsilon=0.05$. The agreement is good.

Determination of $n_{\min }$ is essentially equivalent to the previous and rests on departure $\epsilon^{(N)}$, shown in Fig. 10(a) at several $n$. For $n$ approaching unity (the range of $n_{\min }$ ) computations reveal the departure scales as $\epsilon^{(N)} \propto(n-1)$, implying a local universal scaling function $H_{*}(n)=(n-1) /\left(n_{*}-1\right)$. This, similarly as for $D_{\min }$ and $D_{\max }$, readily leads to

$$
\begin{aligned}
& \frac{n_{\min }-1}{n_{*}-1}=\frac{\epsilon}{\epsilon_{*}^{(N)}} \Rightarrow n_{\min }(D)=1-\frac{\left(1-n_{*}\right) \epsilon}{\epsilon_{*}^{(N)}(D)}, \\
& \epsilon_{*}^{(N)}(D)=\epsilon^{(N)}\left(n_{*}, D\right),
\end{aligned}
$$

as the expression for the threshold $n_{\min }$, with the star again denoting reference quantities. Analogous to $D_{\min }$ and $D_{\max }, n_{\min }$ is also a mapping of its associated reference departure $\epsilon_{*}^{(N)}$. Figure 10(b) shows $n_{\min }$ following (17) $\left(n_{*}\right.$ $=0.75$ ) for $\epsilon=0.05$ (heavy) and $\epsilon=0.1$ (solid), along with direct calculations (symbols) using the direct calculation approach. Again, agreement is good between direct numerical and scaling calculations.

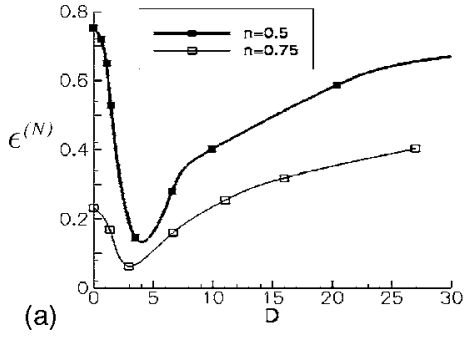

(a)

Downloaded 13 Sep 2007 to 131.155.108.71. Redistribution subject to AIP license or copyright, see http://pof.aip.org/pof/copyright.jsp
FIG. 10. Departure $\epsilon^{(N)}$ at (a) several $n$ and (b) associated threshold $n_{\min }$. The curves in (a) are smooth fits through numerical data (symbols). Threshold $n_{\min }$ at $\epsilon=0.05$ (heavy) and $\epsilon=0.1$ (solid) compared with direct calculations (symbols) (b). (b)

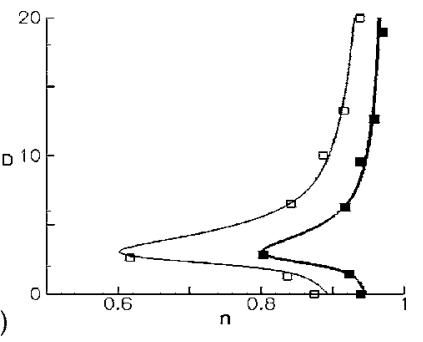



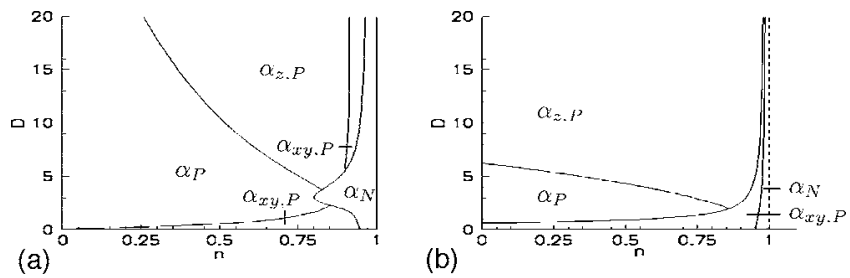

FIG. 11. Flow-model diagram for the power-law model following (a) the velocity-scaling analysis and (b) the viscosity-scaling analysis at $\epsilon=0.05$.

Similarity relations (16) and (17) enable efficient determination of the flow-model diagram for power-law fluids from the reference departures $\epsilon_{*}^{(x y, z)}[$ Fig. 7(a) $]$ and $\epsilon_{*}^{(N)}$ ([Fig. 10(a)]. Figure 11 gives the flow-model diagram attained with the velocity-scaling analysis [Fig. 11(a)] in comparison to that attained through the viscosity-scaling analysis [Fig. 11(b)]. Both diagrams are in qualitative agreement: the location and extent of the ROVs and trends of the thresholds are essentially similar. This establishes, first, the essential consistency between the viscosity-scaling and the velocityscaling approaches, and, second, provides strong evidence for the general validity of the flow-model decomposition.

However, two notable discrepancies exist. The first discrepancy is that in the velocity-scaling diagram the ROVs of the $D \rightarrow 0\left(\alpha_{x y}\right)$ and $D \rightarrow \infty\left(\alpha_{z}\right)$ approximations to the $2.5 \mathrm{D}$ flow model are considerably smaller compared to those in the viscosity-scaling diagram. This implies the viscosityscaling analysis overestimates the ROVs of these simplified flow models. The second discrepancy is the protuberance in the ROV of the Newtonian limit $\left(\alpha_{N}\right)$ in the left diagram (velocity-scaling approach), which stems from the local dip in the departure $\epsilon^{(N)}$ at $D \approx 3$ [Fig. 10(a)]. In this dip $\mathbf{u}$ for each $n$ approaches sufficiently close to unity that the Newtonian limit is within the tolerance $\epsilon$ : $\mathbf{u}$ passes through an intermediate "Newtonian stage" while switching between the limits $D \rightarrow 0$ and $D \rightarrow \infty$. During this Newtonian stage however, the viscosity difference is always above the threshold and so never falls within the Newtonian limit. Figure 12 shows the state at the dip for the axial velocity (a) $u_{z}$ and (b) the viscosity for $n=0.75$, showing the simultaneous approximately Newtonian $\mathbf{u}$ and the significantly non-Newtonian $\alpha$ that characterizes this flow stage.

\section{B. Flow patterns corresponding to the flow-model decomposition}

This section studies the flow patterns corresponding to the flow-model decomposition. Considered, first, is a representative flow field in order to illustrate its typical appear- ance and, second, the changes to the flow field brought on by variation in flow ratio $(D)$ and non-Newtonian departures $(\tau$ and $n$ ) from a Newtonian baseline.

For a representative flow field we take that of the powerlaw case at $n=0.5$ and $D=1.43$. Its relevant flow quantities are given in Fig. 13. The top row shows the transverse flow $\boldsymbol{u}_{x y}$ in terms of its streamline portrait [Fig. 13(a)] and velocity magnitude $\left|\mathbf{u}_{x y}\right|$ [Fig. 13(b)]. Figures 13(a) and 13(b) show the transverse circulation set up by the moving boundary, the large velocities occurring predominantly near the moving boundary, and a stagnation point $\mathbf{u}_{x y}=\mathbf{0}$. The axial flow $u_{z}$ has a nearly axisymmetric shape, almost a Poiseuille profile [Fig. 13(c)]. The viscosity $\alpha$ varies moderately, save near the stagnation point. The viscosity peak near the stagnation point comes from the local vanishing of the shear rate near the stagnation point. The power-law flow field in Fig. 13 is representative of that for any Herschel-Bulkley fluid in this geometry. Computations confirm that all flow fields are qualitatively similar to Fig. 13; changes brought on by variation of the control parameters are, though typically substantial, purely quantitative, consistent with the self-similarity found in Sec. VI A.

The quantitative changes to the base flow from variation of $D$ are shown in Fig. 14. For increasing $D$ the progression of the corresponding flow field (heavy) with its $D \rightarrow 0$ (dashed) and $D \rightarrow \infty$ (dotted) limits $\left(u_{z}:\right.$ top; $u_{\theta}$ : center; $\alpha$ : bottom). The flow field evolves from its $D \rightarrow 0$ state (ROV $\alpha_{x y}$ ) to its $D \rightarrow \infty$ state ( $\operatorname{ROV} \alpha_{z}$ ) in accordance with the scaling prediction. The $D$ values from left-to-right fall within ROV $\alpha_{x y}(D=0.63), \operatorname{ROV} \alpha(D=3.54)$ and $\operatorname{ROV} \alpha_{z}(D$ $=9.95)$, the three distinct regimes in the $D$ direction of the flow-model diagram. The transverse flow $\left(u_{\theta}\right.$; center row in Fig. 14) has only marginal dependence upon $D$; moreover, the shape of the $u_{\theta}$ profiles underscores the previous finding that significant transverse fluid motion is confined to the boundary layer at the moving wall. The axial flow $u_{z}$ (top row) and viscosity $\alpha$ (bottom row) vary considerably with varying $D$, on the other hand. The peaks in the $\alpha$ profiles in the $D \rightarrow 0$ and $D \rightarrow \infty$ limits signify locally unbounded viscosity due to vanishing shear rate caused by vanishing $u_{\theta}(D \rightarrow 0)$ and $d u_{z} / d r(D \rightarrow \infty)$. This implies local solid-like flow and explains the tendency to local flattening of the $u_{z}$ profile near those peaks in the limits $D \rightarrow 0$ [Fig. 14(a)] and $D \rightarrow \infty$ [Fig. 14(c)].

The effect of shear thinning upon the flow dynamics is demonstrated for power-law fluids. Figure 15 shows the changes in flow field ( $\alpha$ : left; $u_{z}$ : center; $u_{\theta}$ : right) caused by increasing departures from the Newtonian baseline $(n=1)$ in the $D \rightarrow 0$ (top) and $D \rightarrow \infty$ (bottom) limits. Considered are
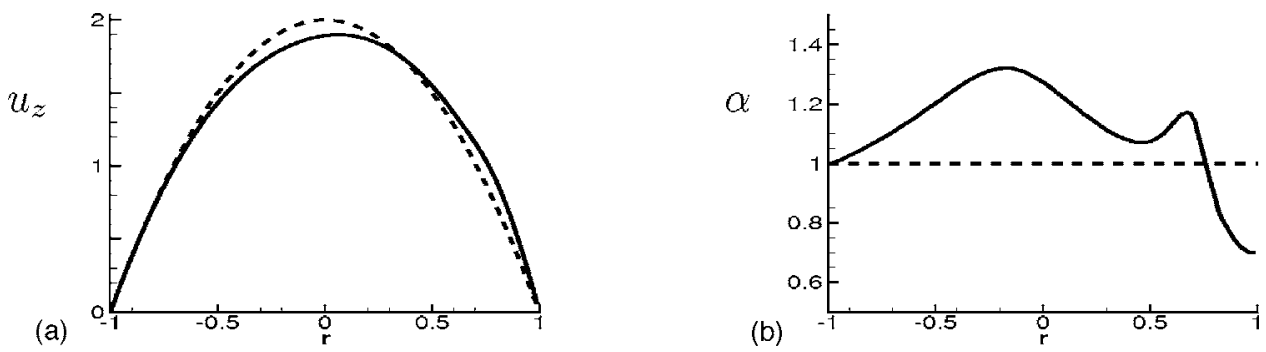

FIG. 12. Intermediate Newtonian stage for the velocity $\mathbf{u}$ at $n=0.75$. Shown are the axial velocity $u_{z}$ and viscosity $\alpha$ at $D \approx 3$ (solid) and the respective Newtonian limits (dashed) on the symmetry axis $\theta=\Delta / 2$. 


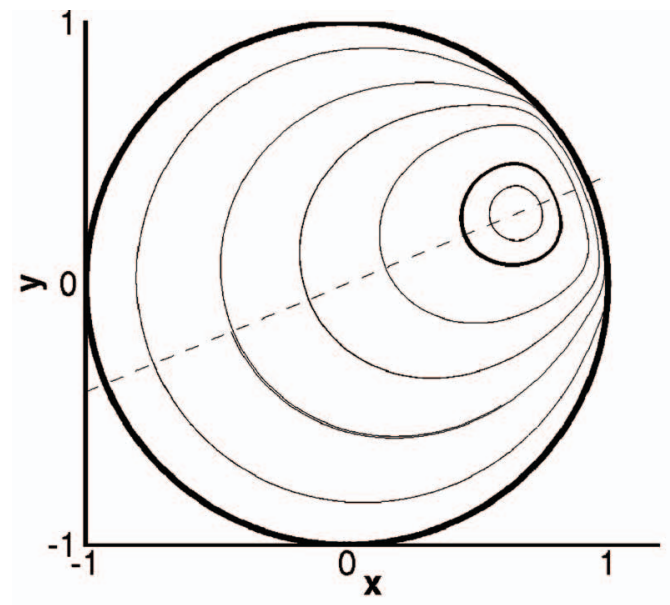

a) Transverse streamline portrait.
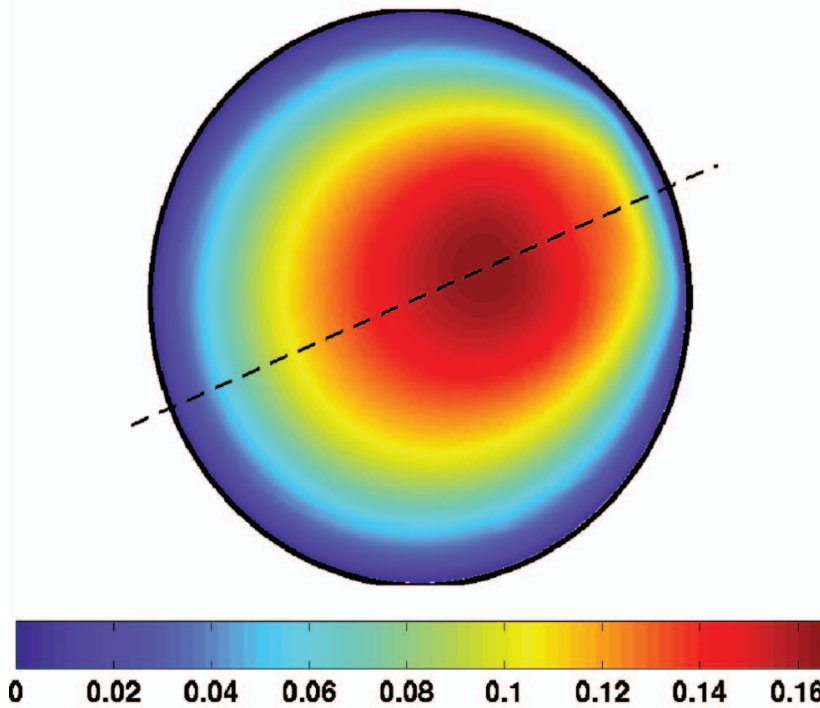

c) Axial velocity $u_{z}$
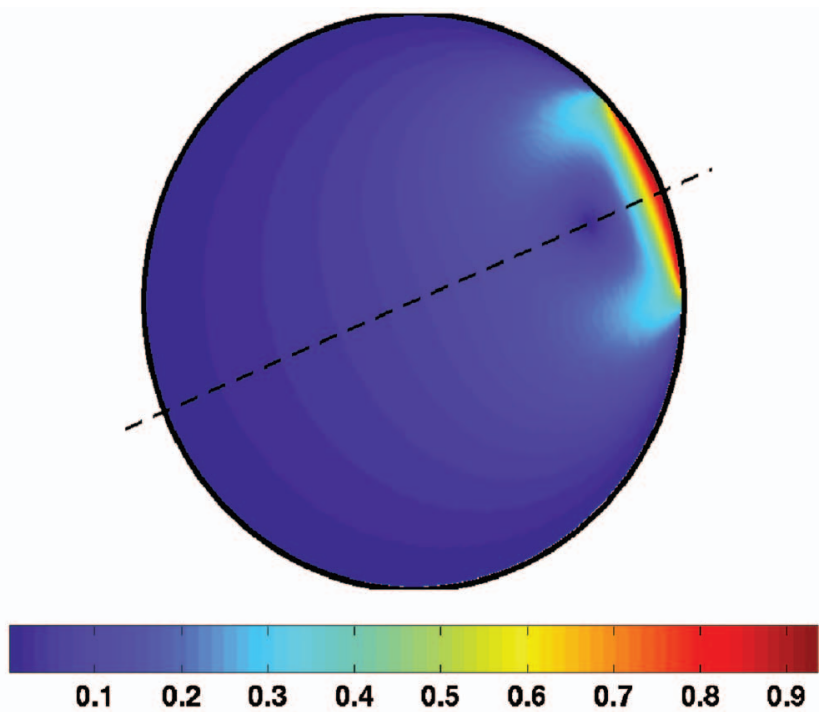

b) Transverse velocity $\left|\boldsymbol{u}_{x y}\right|$.
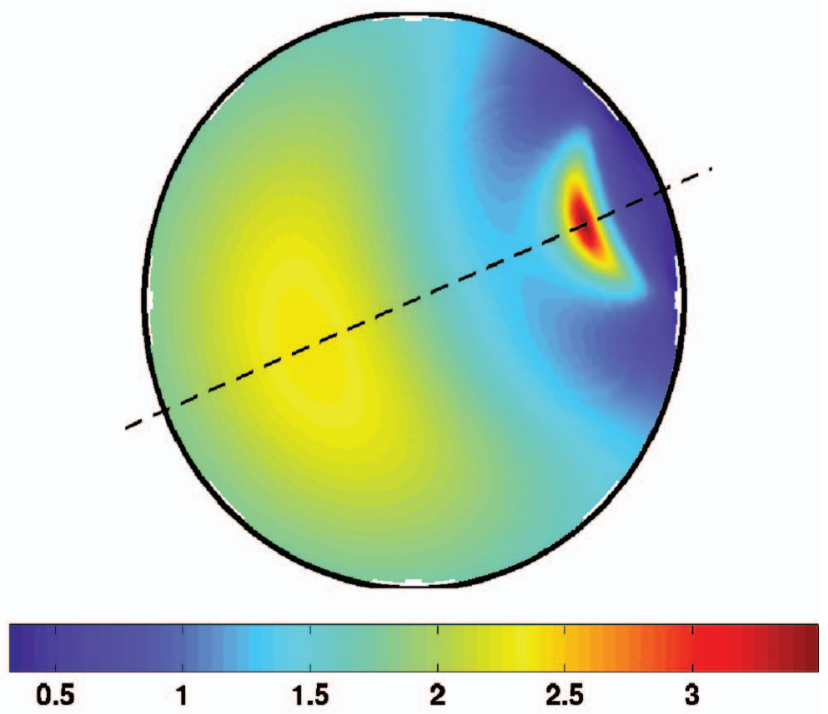

d) Viscosity $\alpha$.

FIG. 13. (Color) Typical flow field in the RAM. Shown is the power-law case for $n=0.5$ and $D=1.43$ The dashed line is the symmetry axis $\theta=\Delta / 2$. The window sits on the right end of the symmetry axis.

the non-Newtonian cases $n=0.25$ and $n=0.5$, which lie well outside the Newtonian regime for any $D$ (see Fig. 11). In particular the profiles of the viscosity $(\alpha)$ and the axial velocity $\left(u_{z}\right)$ show that the local solid-like region near the stagnation point expands for lower flow index $n$ of the fluid; the viscosity peaks rapidly increase in magnitude and the axial profiles quickly flatten in their proximity with decreasing $n$. For the transverse-flow profiles $\left(u_{\theta}\right)$ this tendency is less pronounced although present. For $u_{\theta}$ this solid-like behavior manifests itself in local solid-body rotation and thus locallylinear profiles. Such local linearization for instance underlies the slightly upward-sloping section of the $u_{\theta}$ profile centered on $r=0$ at $n=0.25$ in the limit $D \rightarrow \infty$ [Fig. 15(f)], which coincides with the radius-range occupied by the viscosity peak at $n=0.25$ in Fig. 15(d). Important to note is that the tendency toward solid-like behavior is strongest close to the limits $D \rightarrow 0$ and $D \rightarrow \infty$. In these regimes the viscosity peaks are (nearly) unbounded, whereas outside these limits the viscosity has a bounded peak (see e.g., Fig. 14). Moreover, just as for $D$, the effect of shear-thinning variation is restricted mainly to $\alpha$ and $u_{z}$.

The effect of yielding is demonstrated in the case of Bingham fluids. The changes in flow field brought on by increasing departures from the Newtonian limit $(\tau=0)$ are given in Fig. 16 ( $\alpha$ : left; $u_{z}$ : center; $u_{\theta}:$ right) for the $D \rightarrow 0$ limit. The chosen yield stresses fall within the essentially 

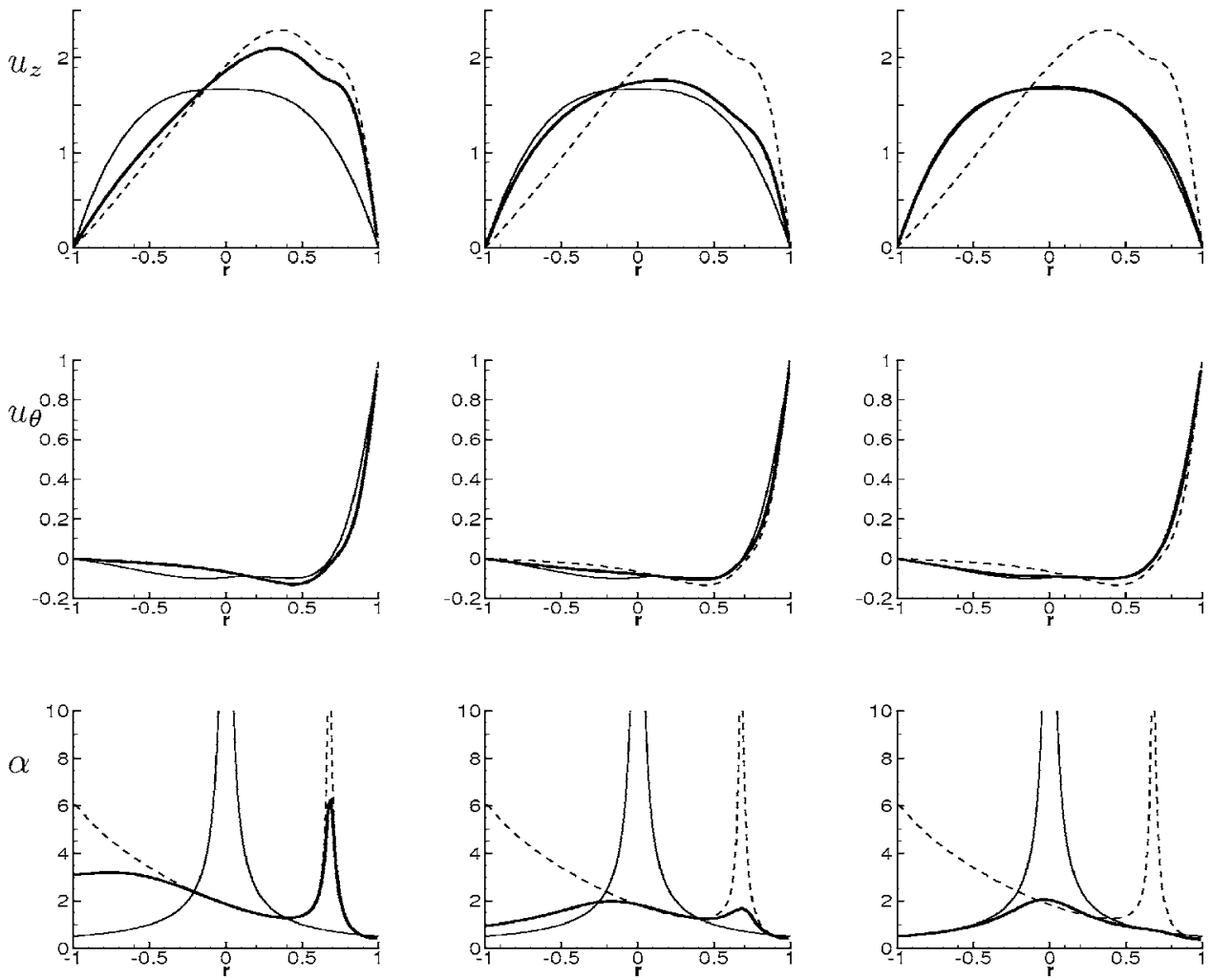

FIG. 14. Limit behavior in $D$ for power-law fluids at $n=0.5$. Shown are the progressions of the axial $\left(u_{z}\right.$; top) and transverse $\left(u_{\theta}\right.$; center) flows and the viscosity ( $\alpha$; bottom) on the symmetry axis $\theta=\Delta / 2$ (heavy) from limit $D \rightarrow 0$ (dashed) to limit $D \rightarrow \infty$ (solid) for increasing $D$.

non-Newtonian regime (Fig. 5). In particular the development of the viscosity and axial flow for increasing yield stress clearly resembles that caused by increased shear thinning (Fig. 15; top row). The viscosity peaks grow in magnitude and the axial profiles flatten in their proximity with increasing $\tau$. Again the transverse flow exhibits only marginal dependence upon variations in $\tau$. A minor quantitative difference with the power-law case exists in that here the viscosity peaks at both the left wall and to the right of the centerline tend to be more localized than their power-law counterparts. The striking resemblance between the changes in flow patterns caused by shear thinning and yielding nonetheless strongly suggests both phenomena have comparable impact upon the flow dynamics, despite fundamentallydifferent underlying rheological mechanisms. For example, Fig. 17 further demonstrates that fundamentally-different fluid rheologies (Bingham: $\tau=1$; HB: $\tau=0.2, n=0.5$ ) have near-identical flow fields. Combined moderate yielding and
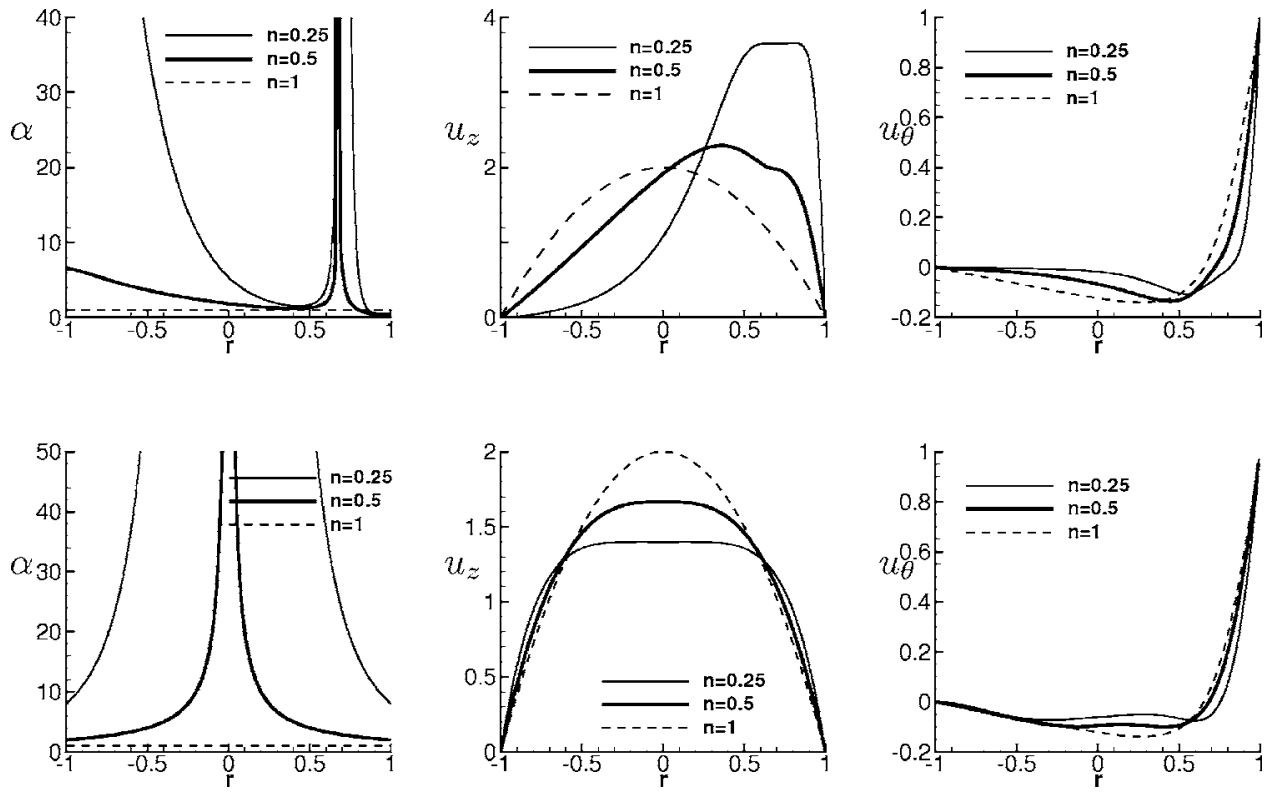

FIG. 15. Non-Newtonian departures from the Newtonian baseline $(n=1)$ for power-law fluids. Shown are the changes in pattern on the symmetry axis $\theta=\Delta / 2$ for the axial $\left(u_{z}\right)$ and transverse $\left(u_{\theta}\right)$ flows and the viscosity $(\alpha)$ for limit $D \rightarrow 0$ (top row) and limit $D \rightarrow \infty$ (bottom row). 

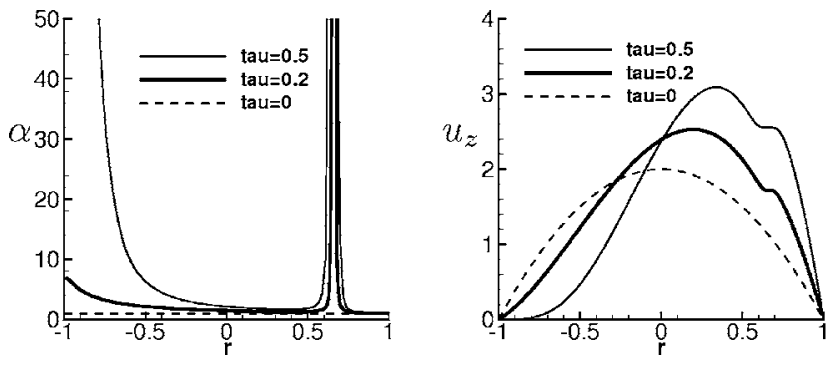

shear thinning thus is, as to resulting flow field, comparable to either strong yielding or shear thinning. This implies both the power-law and Bingham models provide, to first approximation, an adequate description of non-Newtonian phenomena.

\section{CONCLUSIONS}

We have presented a theoretical and numerical analysis on the scaling properties of reoriented duct flows of generalized Newtonian fluids. Reoriented duct flows consist of a superposition of a uniaxial flow and an axial-wise reoriented transverse flow. The non-Newtonian behavior has been described by the Herschel-Bulkley rheology model, which incorporates pseudo-plastic and yield effects. Further, the study has been restricted to noninertial flows described by a cellular model that requires resolution of the flow field in the cross section of just one cell.

The duct flows exhibit limit behavior in the sense that they become (nearly) independent of one or more of their control parameters and reduce to simpler forms in specific regions of the parameter space of $n$ (flow index) and $\tau$ (nondimensional yield stress or Bingham number) and the dynamical parameter $D$ (ratio of characteristic transverse and axial velocities). Various limits of these parameters identified flow states and flow regimes via a scaling analysis to decompose the generic flow field into a set of fundamental flow states and associated flow regimes. As the scaling is universal, the flow-model decomposition holds for all duct flows.

Application of the flow-model decomposition requires relaxation of the limit flows via an imposed tolerance of deviation from the full cellular flow model in order to attain finite ROVs for the simplified flow models. The scaling analysis also determines finite ROVs for the flow models that are compiled into a flow-model diagram to provide a visual representation of the flow-model decomposition and the fundamental flow states of reoriented duct flows (Fig. 4). This combination of ROVs demarcates ten distinct regions and implies reoriented duct flows have ten fundamental flow states, in complexity ranging from the full Herschel-Bulkley state down to the Newtonian baseline (Table I).

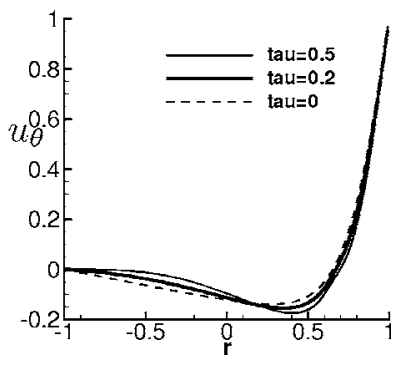

FIG. 16. Non-Newtonian departures from the Newtonian baseline $(\tau=0)$ for Bingham fluids. Shown are the changes in pattern on the symmetry axis $\theta=\Delta / 2$ for the axial $\left(u_{z}\right)$ and transverse $\left(u_{\theta}\right)$ flows and the viscosity $(\alpha)$ in the limit $D \rightarrow 0$.
Tests of the flow-model decomposition have been performed by computation on a specific flow, the RAM flow, which is representative of generic reoriented duct flows. The flow-model diagram was determined for the power-law limit of the Herschel-Bulkley rheology model using numerical velocity fields. This analysis disclosed a self-similarity in $n$ of the average velocity field that enabled efficient and robust determination of the bounds of the several ROVs via a similarity-based scaling approach. The flow-model diagram found with this velocity-scaling approach was in good qualitative agreement with that attained via the viscosity-scaling analysis (Fig. 11). This established, first, the essential consistency between viscosity-scaling analysis and the velocityscaling approach and, second, provided strong evidence as to the general validity of the generic flow-model decomposition based upon the viscosity-scaling analysis. Two notable discrepancies were found between the two approaches. First, the ROVs of the limit flows found with the velocity-scaling analysis are substantially smaller compared to those found with the viscosity-scaling analysis. This implies in the practical context of mixing flows (to which the properties of the velocity field are key) the viscosity-scaling analysis overestimates the ROVs of these limit flows. Second, the velocityscaling analysis revealed that moderately non-Newtonian rheologies admit flow fields with an essentially nonNewtonian viscosity yet a (within acceptable tolerances) Newtonian velocity field (Fig. 12). This means for mixing applications the ROV of the Newtonian limit is larger than that predicted by the viscosity-scaling analysis.

Flow patterns corresponding to the flow-model decomposition were determined for the specific case of the RAM flow. The study revealed the flow patterns are-at any rheology-qualitatively similar and invariably symmetric about the symmetry axis of the transverse forcing. Despite the qualitative invariance of the flow field, quantitative differences brought on by variation of control parameters could be substantial.

The response of the flow field to variations in flow ratio $D$ are also similar for all rheologies. Increasing $D$ from limit $D \rightarrow 0$ to limit $D \rightarrow \infty$ causes shifting of the region of solid-
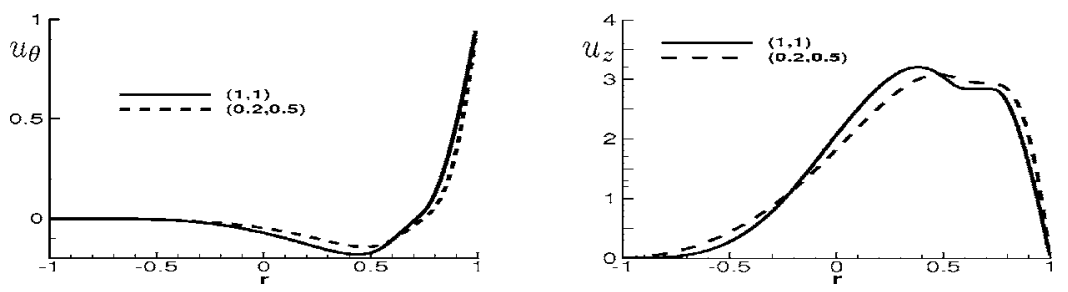

FIG. 17. Similarity in flow patterns despite significant differences in rheology. Shown are the axial $\left(u_{z}\right)$ and transverse $\left(u_{\theta}\right)$ flows on the symmetry axis $\theta=\Delta / 2$ in the limit $D \rightarrow 0$. Given numbers are yield-stress flowindex settings: $(\tau, n)$. 

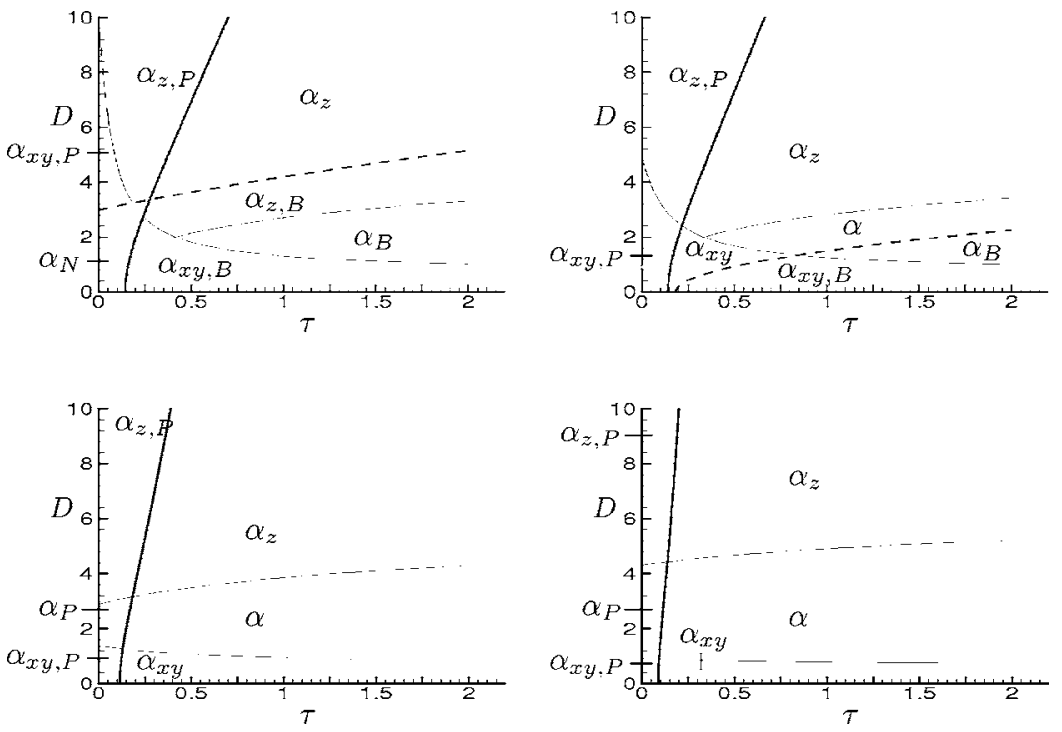

FIG. 18. Isoplanes of the flow index $n$ in the flowmodel diagram (Fig. 4). like motion (at the viscosity peak) from the center of circulation of the transverse flow to the origin. For the viscosity and axial flow this transforms the essentially asymmetric state at $D \rightarrow 0$ into the axisymmetric state at $D \rightarrow \infty$. Noteworthy is that significant variation is restricted mainly to the viscosity and the axial flow; the transverse flow varies but marginally.

Increasingly non-Newtonian behavior introduced through increased shear thinning (flow index $n$ ) and/or increased yield stress $(\tau)$ amplifies the non-Newtonian features of the flow (viscosity peak; local solid-like behavior) that are present at a given flow ratio $D$ but does not cause fundamental changes in flow field. This is consistent with the selfsimilarity of the average flow field. The changes brought on by shear thinning and yielding are qualitatively similar and differ only in that yielding leads to somewhat more compact regions of solid-like motion compared to the shear-thinning case. This suggests both phenomena have comparable impact upon the flow dynamics, despite fundamentally different underlying rheological mechanisms, which is consistent with the fact that the flow-model diagrams of the power-law [Fig. 5(a)] and Bingham [Fig. 5(b)] limits are qualitatively similar. Tests reveal that flow fields with either strong shear-thinning or strong yielding rheologies are comparable to those found for moderately shear-thinning/yielding rheologies (Fig. 17) and thus imply that either the power-law or the Bingham model provide a good (first order) description of the nonNewtonian Herschel-Bulkley rheology. This strong analogy between shear thinning and yielding indirectly extends the validity of the flow-model decomposition established in Sec. VI A for the power-law case to the full Herschel-Bulkley rheology model. Further, the good agreement between the flow-model decomposition predicted by the generic viscosity-scaling analysis and that determined for the representative test flow provides strong evidence as to the validity of the viscosity-scaling analysis and the flow-model decomposition for generic reoriented duct flows.

The scaling analysis and flow-model decomposition of reoriented duct flows of generalized Newtonian fluids presented here may find application in a wide range of situations. The cellular approach admits application to both reoriented and nonreoriented duct flows. The analysis offers a
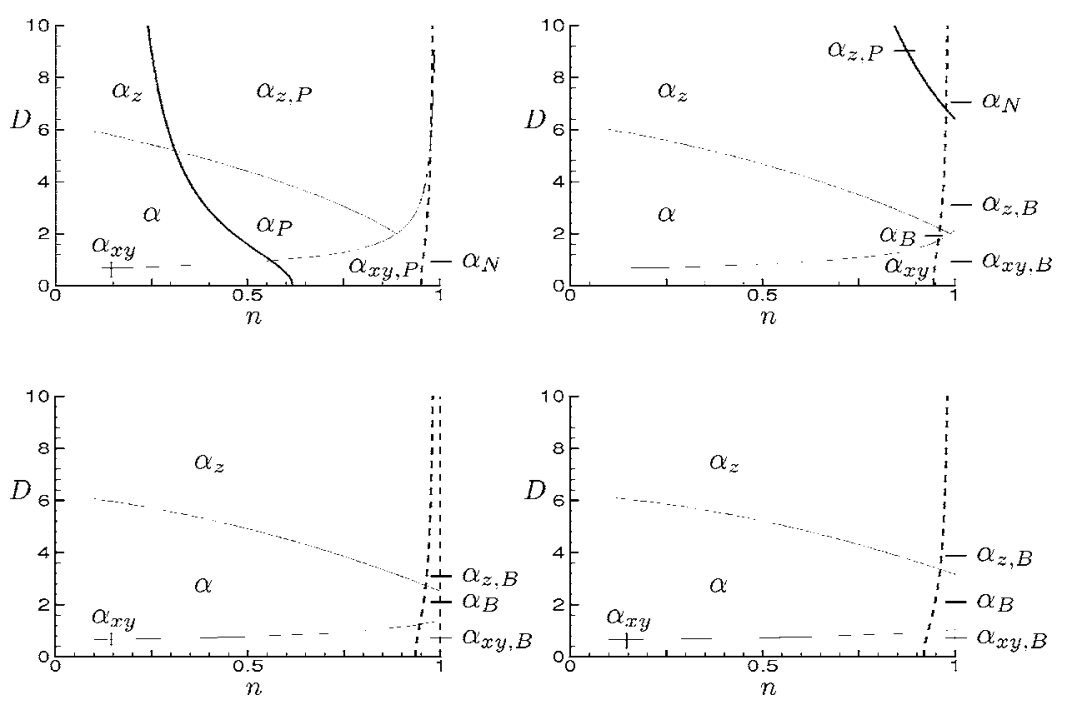

FIG. 19. Isoplanes of the yield stress $\tau$ in the flowmodel diagram (Fig. 4). 
way to systematically isolate the dominant rheological and/or dynamical features in a given configuration as a function of its control parameters. This enhances insight into the physics of duct flows and also admits robust modeling and efficient yet reliable examination. Moreover, the analysis enables systematic up/down scaling of non-Newtonian (reoriented) duct flows with length scales ranging from small-scale microfluidic applications to large-scale industrial applications and a wide range of rheologies to representative configurations. This systematic rescaling to representative cases may find application in the design of laboratory experiments and in design codes of industrial devices involving continuous flows of non-Newtonian fluids.

\section{APPENDIX: ISOPLANES IN THE FLOW-MODEL DIAGRAM}

\section{See Figs. 18 and 19.}

${ }^{1}$ R. Sluijters, "Het principe van de multiflux menger," in De Ingenieur, Chemische Techniek 3, (1965), Vol. 77 (in Dutch).

${ }^{2}$ M. H. Pahl and E. Muschelknautz, "Static mixers and their applications," Chem.-Ing.-Tech. 52, 285 (1980).

${ }^{3}$ G. Metcalfe, M. Rudman, A. Brydon, L. Graham, and R. Hamilton, "Composing chaos: an experimental and computational study of an open duct mixing flow," AIChE J. 52, 9 (2006).

${ }^{4}$ D. M. Hobbs, P. D. Swanson, and F. J. Muzzio, "Numerical characterization of low Reynolds number flow in the Kenics static mixer," Chem. Eng. Sci. 53, 1565 (1998).

${ }^{5}$ D. V. Khakhar, J. G. Franjione, and J. M. Ottino, "A study of chaotic mixing in deterministic flows, the partitioned pipe mixer," Chem. Eng. Sci. 42, 2909 (1987).

${ }^{6} \mathrm{H}$. A. Kusch and J. M. Ottino, "Experiments on mixing in continuous chaotic flows," J. Fluid Mech. 236, 319 (1992).

${ }^{7}$ F. H. Ling, "Chaotic mixing in a spatially periodic continuous mixer," Phys. Fluids A 5, 2147 (1993).

${ }^{8}$ V. V. Meleshko, O. S. Galaktionov, G. W. M. Peters, and H. E. H. Meijer, "Three-dimensional mixing in Stokes flow: the partitioned-pipe mixer problem revisited," Eur. J. Mech. B/Fluids 18, 783 (1999).

${ }^{9}$ Y. Mizuno and M. Funakoshi, "Chaotic mixing due to a spatially periodic three-dimensional flow," Fluid Dyn. Res. 31, 129 (2002).

${ }^{10}$ J. C. van der Hoeven, R. Wimberger-Friedl, and H. E. H. Meijer, "Homogeneity of multilayers produced with a static mixer," Polym. Eng. Sci. 41, 32 (2001).
${ }^{11}$ J. E. Visser, P. F. Rozendal, H. W. Hoogstraten, and A. A. C. M. Beenackers, "Three-dimensional numerical simulation of flow and heat transfer in the Sulzer SMX static mixer," Chem. Eng. Sci. 54, 2491 (1999).

${ }^{12}$ K. D. P. Nigam and E. B. Nauman, "Residence time distribution of powerlaw fluids in motionless mixers," Can. J. Chem. Eng. 63, 519 (1985).

${ }^{13}$ Z. Kemblowski and P. Pustelnik, 1988 "Residence time distribution of a power-law fluid in Kenics static mixers," Chem. Eng. Sci. 43, 473 (1988).

${ }^{14}$ P. Fast, L. Kondic, M. J. Shelley, and P. Palffy-Muhoray, "Pattern formation in non-Newtonian Hele-Shaw flow," Phys. Fluids 13, 1191 (2001).

${ }^{15}$ R. I. Tanner, Engineering Rheology (Clarendon, Oxford, 1985).

${ }^{16}$ G. I. Barenblatt, Scaling, Self-Similarity, and Intermediate Asymptotics (Cambridge University Press, Cambridge, 1996).

${ }^{17}$ Any method of axial forcing is allowed (pressure-driven, electro-osmotic, etc.), provided it remains constant in the axial direction, the analysis is similar to the pressure-driven case.

${ }^{18}$ Realistic duct flows may involve cell-wise $z$-dependent forcings. Reconciliation with the present duct flows is-at least qualitatively-possible via an appropriately chosen frame of reference.

${ }^{19}$ Note that in cases where the axial flow is not strictly continuous across axial cell boundaries, the $2.5 \mathrm{D}$ approach will locally violate incompressibility at cell boundaries. However, this does not appear to pose a problem with getting predictions accurate against experiment. ${ }^{3}$

${ }^{20}$ Component expansions of the nondimensional contraction of the $2.5 \mathrm{D}$ flow model:

$$
\begin{aligned}
& \underbrace{\mathbf{D}: \mathbf{D}=\left(\frac{\partial u_{x}}{\partial x}\right)^{2}+\left(\frac{\partial u_{y}}{\partial y}\right)^{2}+\frac{1}{2}\left(\frac{\partial u_{x}}{\partial y}+\frac{\partial u_{y}}{\partial x}\right)^{2}}_{=\mathbf{D}_{x y}: \mathbf{D}_{x y}}+\frac{D^{2}}{2}[\underbrace{\left(\frac{\partial u_{z}}{\partial x}\right)^{2}+\left(\frac{\partial u_{z}}{\partial y}\right)^{2}}_{=\left|v u_{z}\right|^{-}}] \\
& \mathbf{D}_{x y}: \mathbf{D}_{x y}=\left(\frac{\partial u_{r}}{\partial r}\right)^{2}+\left(\frac{u_{r}}{r}+\frac{1}{r} \frac{\partial u_{\theta}}{\partial \theta}\right)^{2}+\frac{1}{2}\left(\frac{\partial u_{\theta}}{\partial r}-\frac{u_{\theta}}{r}+\frac{1}{r} \frac{\partial u_{r}}{\partial \theta}\right)^{2}
\end{aligned}
$$

${ }^{21}$ A. Souvaliotis, S. C. Jana, and J. M. Ottino, "Potentialities and limitations of mixing simulations," AIChE J. 41, 1605 (1995).

${ }^{22}$ In the limit $D \rightarrow \infty$, constant $C_{p}$ and homogeneous boundary conditions imply $u_{z}=u_{z}(r)$ and $\alpha=\alpha_{z}(r)$, for which analytical solutions to the $2.5 \mathrm{D}$ model are given in Eirich (Ref. 23). For the Newtonian limit, analytical expressions for $\mathbf{u}_{x y}$ are given in Hwu et al. (Ref. 24); the axial flow coincides with the Poiseuille profile $u_{z}=2\left(1-r^{2}\right)$.

${ }^{23}$ F. R. Eirich, Rheology: Theory and Applications Volume 1 (Academic, New York, 1956).

${ }^{24}$ T-Y. Hwu, D. L. Young, and Y-Y. Chen, 1997 "Chaotic advection for Stokes flow in a circular cavity,” J. Eng. Mech. 123, 774 (1997).

${ }^{25}$ E. Kreyszig, Advanced Engineering Mathematics (Wiley, Chichester, 1999).

${ }^{26} \mathrm{~A}$ third-order polynomial expansion in $n$, incorporating $H_{*}\left(n_{*}\right)=1$ and $H_{*}(1)=0$ 